\title{
Tauroursodeoxycholic bile acid arrests axonal degeneration by inhibiting the unfolded protein response in $\mathrm{X}$-linked adrenoleukodystrophy
}

\author{
Nathalie Launay $^{1,2} \cdot$ Montserrat Ruiz $^{1,2} \cdot$ Laia Grau $^{1,2}$ - Francisco J. Ortega ${ }^{1,2}$ - Ekaterina V. Ilieva ${ }^{1,2,3}$ • \\ Juan José Martínez ${ }^{1,2} \cdot$ Elena Galea $^{4,5} \cdot$ Isidre Ferrer $^{6,7,8} \cdot$ Erwin Knecht $^{9,10} \cdot$ Aurora Pujol $^{1,2,5}$ D \\ Stéphane Fourcade ${ }^{1,2}$
}

Received: 12 April 2016 / Revised: 9 December 2016 / Accepted: 9 December 2016 / Published online: 21 December 2016

(C) The Author(s) 2016. This article is published with open access at Springerlink.com

\begin{abstract}
The activation of the highly conserved unfolded protein response (UPR) is prominent in the pathogenesis of the most prevalent neurodegenerative disorders, such as Alzheimer's disease (AD), Parkinson's disease (PD) and amyotrophic lateral sclerosis (ALS), which are classically characterized by an accumulation of aggregated or misfolded proteins. This activation is orchestrated by three endoplasmic reticulum (ER) stress sensors: PERK, ATF6 and IRE1. These sensors transduce signals that induce the expression of the UPR gene programme. Here, we first identified an early activator of the UPR and investigated the role of a chronically activated UPR in the pathogenesis of X-linked adrenoleukodystrophy (X-ALD), a neurometabolic disorder that is caused by $\mathrm{ABCD} 1$ malfunction;
\end{abstract}

A. Pujol and S. Fourcade contributed equally.

Electronic supplementary material The online version of this article (doi:10.1007/s00401-016-1655-9) contains supplementary material, which is available to authorized users.

Aurora Pujol

apujol@idibell.cat

Stéphane Fourcade

sfourcade@idibell.cat

$1 \quad$ Neurometabolic Diseases Laboratory, Institut de Neuropatologia de Bellvitge, IDIBELL, Gran Via, 199, L'Hospitalet de Llobregat, 08908 Barcelona, Spain

2 CIBERER U759, Center for Biomedical Research on Rare Diseases, Barcelona, Spain

3 Present Address: Global data, London, UK

4 Institute of Neurosciences, Universitat Autònoma de Barcelona, Barcelona, Spain

5 Catalan Institution of Research and Advanced Studies (ICREA), Barcelona, Spain
ABCD1 transports very long-chain fatty acids (VLCFA) into peroxisomes. The disease manifests as inflammatory demyelination in the brain or and/or degeneration of corticospinal tracts, thereby resulting in spastic paraplegia, with the accumulation of intracellular VLCFA instead of protein aggregates. Using X-ALD mouse model $\left(A b c d 1^{-}\right.$ and $A b c d 1^{-} / A b c d 2^{-/-}$mice) and X-ALD patient's fibroblasts and brain samples, we discovered an early engagement of the UPR. The response was characterized by the activation of the PERK and ATF6 pathways, but not the IRE1 pathway, showing a difference from the models of $\mathrm{AD}, \mathrm{PD}$ or ALS. Inhibition of PERK leads to the disruption of homeostasis and increased apoptosis during ER stress induced in X-ALD fibroblasts. Redox imbalance appears to be the mechanism that initiates ER stress in X-ALD. Most importantly, we demonstrated that the bile acid tauroursodeoxycholate (TUDCA) abolishes UPR activation, which results in improvement of axonal degeneration and its associated locomotor impairment in $A b c d 1^{-} / A b c d 2^{-/-}$mice.

6 Institute of Neuropathology, Pathologic Anatomy Service, IDIBELL, Barcelona, Spain

7 Department of Pathology and Experimental Therapeutics, University of Barcelona, 08908, L'Hospitalet de Llobregat, Barcelona, Spain

8 CIBERNED, Center for Biomedical Research on Neurodegenerative Diseases, Barcelona, Spain

9 Laboratory of Cellular Biology, Centro de Investigación Príncipe Felipe, 46012 Valencia, Spain

10 CIBERER U721, Center for Biomedical Research on Rare Diseases, Valencia, Spain 
Altogether, our preclinical data provide evidence for establishing the UPR as a key drug target in the pathogenesis cascade. Our study also highlights the potential role of TUDCA as a treatment for X-ALD and other axonopathies in which similar molecular mediators are implicated.

Keywords Peroxisome · ER stress · UPR .

Oxidative stress - Neurodegeneration - TUDCA ·

Adrenoleukodystrophy

\section{Introduction}

The unfolded protein response (UPR) is a cellular stress reaction of the endoplasmic reticulum (ER) that is caused by defective protein processing $[36,82,100]$. Under physiological conditions, misfolded proteins undergo the ERassociated degradation process (ERAD), whereby the ER degradation-enhancing $\alpha$-mannosidase-like protein (EDEM) forms complexes with other proteins, such as the protein disulphide isomerase (PDI) and the glucose-regulated protein 78 (GRP78; also known as Bip) and glucoseregulated protein 94 (GRP94) chaperones, to guide the translocation of misfolded proteins back to the cytosol. The misfolded proteins are then polyubiquitinated for targeted degradation by the proteasome. Through this pathway, the so-called UPR is activated by an aberrant accumulation of misfolded or unfolded proteins in the ER compartment due to changes in intra-reticular calcium, altered protein glycosylation, energy deprivation, pathogen infection, expression of folding-defective proteins, or changes in redox status. The UPR is distinguished by the action of three ER-located transmembrane receptors (protein kinase RNA-like endoplasmic reticulum kinase (PERK), activating transcription factor (ATF6) and inositol requiring kinase (IRE1)), which regulate these events in concert.

Thus, in mammalian cells all three pathways, which are the so-called ER stress sensors, are involved in the UPR and have partially overlapping roles as described below [36, 82, 100]. After ER stress, unfolded proteins accumulate and PERK, along with additional proteins, attenuates mRNA translation, thereby preventing protein overload into the already stressed ER compartment. This translational attenuation is mediated by the phosphorylation of eukaryotic translation initiation factor 2 subunit $\alpha$ (eIF2 $\alpha$ ), which is essential for adaptation to cellular stress through the integrated stress response (ISR) process. The phosphorylation of eIF $2 \alpha$ enables the preferential translation of UPR-dependent genes, such as the transcription factor ATF4, which drives the transcription of several critical genes, including the pro-apoptotic factor CHOP [36, 82, 100].
ATF6 is a transcription factor that translocates to the Golgi compartment upon ER stress, where it is cleaved [105]. Cleaved ATF6 then enters the nucleus to activate target genes, such as GRP78, GRP94, PDI and CHOP.

Upon activation, IRE1, which is a transmembrane kinase/endonuclease, initiates the splicing of the X-boxbinding protein $1(X B P 1)$ mRNA. Spliced XBPI $(X B P 1 s)$ mRNA leads to the transcription of several genes that are involved in the UPR and ERAD to restore protein homeostasis and promote cytoprotection $[36,82,100]$. In conclusion, the first outcome of the UPR is protective as it helps maintain homeostasis. However, if the stress is not successfully resolved, the UPR can finally trigger apoptosis. The same pathways can be anti- or pro-apoptotic depending on the intensity, duration and cellular context of the UPR activation $[36,44,78,82,100]$.

Unfolded protein response activation has classically been associated with several neurodegenerative and metabolic diseases that are characterized by protein aggregates, including Alzheimer's disease (AD) [41, 42, 82], Huntington's disease (HD) [95], amyotrophic lateral sclerosis (ALS) [81], Parkinson's disease (PD) [82], Pick's disease [47], and argyrophilic grain disease [46]. Despite several studies that support the interconnected nature of UPR activation, only a few reports have addressed the role of the ER and UPR in diseases that are driven by redox and lipid dyshomeostasis instead of protein misfolding [61, 91]. We therefore sought to explore the role of ER stress in a paradigmatic disease of oxidative stress and lipid rather than protein accumulation: X-linked adrenoleukodystrophy (X-ALD: McKusick no. 300100) [28, 57].

$\mathrm{X}$-ALD is a rare, fatal, neurometabolic disease that is characterized by a striking variation in clinical symptoms, even within the same family. The two main neurological phenotypes include (i) the devastating childhood cerebral form (CCALD), which progresses rapidly and is associated with rampant inflammatory demyelination in the brain, and the more common phenotype of (ii) slowly progressing adult-onset adrenomyeloneuropathy (AMN), which manifests as a distal axonopathy of corticospinal tracts, thereby leading to spastic paraparesis with peripheral neuropathy in some cases [26, 66]. Approximately 35\% of AMN patients develop cerebral demyelination (cAMN) and share the same poor prognosis as children with cerebral ALD. All patients with X-ALD have mutations in the $A B C D 1$ gene in $\mathrm{Xq} 28$, which encodes the peroxisomal adrenoleukodystrophy protein (ALDP or ABCD1) [26, 66]; ALDP transports very long-chain fatty acids (VLCFA) or VLCFA-CoA esters into the peroxisome for degradation by $\beta$-oxidation [93]. Therapeutic options are scarce, and when diagnosed early, the cerebral forms of the disease are only adequately treatable with a bone marrow transplant [1] or, recently, 
haematopoietic stem cell gene therapy [9]. However, no pharmacological treatment has been proven to be beneficial for either form of the disease [6].

The mouse model of X-ALD, the $A b c d 1^{-}$mouse, develops late-onset axonopathy that resembles the most frequent X-ALD phenotype, AMN $[75,76]$. Using this mouse model and patient samples, studies by our laboratory and others have revealed that VLCFA-induced oxidative stress is an early, key pathogenic factor in X-ALD [29, 31, 35, 57, $73,74,85,94]$, although the exact mechanisms by which oxidative stress causes neurodegeneration in X-ALD are not entirely clear.

Oxidative stress and lipid alterations are highly intertwined with ER stress [61, 91, 97, 98]; we thus posited that UPR activation may have a role in the pathogenesis of X-ALD. To test this hypothesis, we determined whether the UPR (i) is activated in brain samples of patients who suffered from the cerebral forms of X-ALD (CCALD and cAMN), the spinal cord from the mouse model of X-ALD and in human X-ALD fibroblasts and (ii) is a causative factor of axonal degeneration, which we determined by assessing the effects of the ER stress inhibitor tauroursodeoxycholic acid (TUDCA) on the late-onset axonopathic phenotype that is exhibited in the X-ALD $\left(\mathrm{Abcdl}^{-}\right.$) $A b c d 2^{-/}$) mouse model.

\section{Materials and methods}

\section{Human samples}

Brain tissue samples from X-ALD patients and agematched controls were obtained from the NIH NeuroBioBank: details are in the supplemental data (Supplemental Table S1).

Primary human fibroblasts were collected from healthy individuals and AMN patients according to the IDIBELL guidelines for sampling, including informed consent from the persons involved or their representatives. Fibroblasts were prepared from skin biopsies (Supplemental Table S2).

\section{X-ALD mice}

Two X-ALD mouse models were used in this study. The first model, the $A b c d 1^{-}$mouse, is used to characterize the biochemical signs as it resembles adult AMN in humans $[59,76]$. These mice exhibit metabolic signs of pathology, including oxidative stress [29] and altered energy homeostasis [34]; however, the first clinical signs of AMN (axonopathy and locomotor impairment) appear at 20 months [76]. The second mouse model has a double gene knockout of both the $A b c d l$ and $A b c d 2$ transporters $\left(A b c d 1^{-} / A b c d 2^{-/-}\right)$and is a more suitable model for assaying therapeutic strategies than $A b c d 1^{-}$mice. Indeed, compared with $A b c d 1^{-}$mice, $A b c d 1^{-} / A b c d 2^{-/}$mice present enhanced VLCFA accumulation in the spinal cord [75], higher levels of oxidative damage to proteins [31], and a more severe AMN-like pathology with an earlier onset at 12 months of age [30, 55, 62, 65, 75].

Male mice used for experiments were of a pure C57BL/6J background. Generation and genotyping for $A b c d 1^{-}$and $A b c d 1^{-} / A b c d 2^{-/-}$mice have been previously described $[59,75,76]$.

For the antioxidant treatment, wild type (WT) $(n=10)$ and $A b c d 1^{-}$mice $(n=20)$ were separated into three different groups at 8 months of age [WT $(n=10), A b c d 1^{-}$ $(n=10)$ and $A b c d 1^{-}$mice treated with a cocktail of antioxidants [Vitamin E (1000 iu/kg) and $\alpha$-lipoic acid $(0.5 \%)$ in AIN-76A chow (Ssniff) and NAC (1\%) in water] for 4 months $\left(A b c d 1^{-}+\right.$Aox $\left.)(n=10)\right][34,56,57]$. At 12 months of age, mice were killed, and tissue samples were collected and snap-frozen in liquid nitrogen.

For the TUDCA treatment, WT $(n=10)$ and $A b c d 1^{-}$ mice $(n=20)$ were separated into three different groups at 9 months of age [WT $(n=10), A b c d 1^{-}(n=10)$ and $A b c d 1^{-}$mice treated with the bile acid TUDCA (Prodotti Chimici e Alimentari SpA, Italy) for 3 months $\left(A b c d 1^{-}+\right.$TUDCA) $\left.(n=10)\right]$. TUDCA was mixed into AIN-76A chow (Ssniff) at $0.4 \% \mathrm{w} / \mathrm{w}$. In addition, WT $(n=15)$ and $A b c d 1^{-} / A b c d 2^{-/-}$mice $(n=30)$ were separated into three different groups at 13 months of age [WT $(n=15), A b c d 1^{-} / A b c d 2^{-/-}(n=15)$ and $A b c d 1^{-} / A b c d 2^{-/-}$ mice treated with TUDCA $(0.4 \% \mathrm{w} / \mathrm{w})$ for 4 months $\left(A b c d 1^{-} / A b c d 2^{-/}+\right.$TUDCA $\left.)(n=15)\right]$. TUDCA had no effect on weight or food intake under either treatment protocol. The dose and duration of TUDCA, whose pharmacokinetics have been extensively studied in rodents [14], was based on pilot studies using APP/PS1 mice and other models [11, 67].

After 4 months of treatment, locomotor experiments were performed for three weeks, one test a week, in order not to exhaust the mice. Animals were maintained under treatment during this period and were killed and processed at the end for immunohistology. For behavioural testing, assessment of hindlimb clasping was done by suspending WT and $A b c d 1^{-} / A b c d 2^{-/-}$mice from their tails until they reached a vertical position, allowing the mice to grab the grill of the lid of the cage with the forelimbs, as adapted from Dumser et al. [19]. The hindlimb reflex was analysed for $10 \mathrm{~s}$ in three consecutive trials separated by 5 -min rest. The hindlimb reflex was scored as described in Supplemental Table S3. The treadmill and bar cross experiments were performed exactly as previously described [57, 64]. Mockand drug-treated mice were simultaneously tested using these three locomotor tests (clasping, treadmill and bar cross) for both genotype and treatment in a blinded manner. 
All methods employed in this study were in accordance with the Guide for the Care and Use of Laboratory Animals published by the US National Institutes of Health (NIH publication No. 85-23, revised 1996) and the ethical committees of IDIBELL and the Generalitat de Catalunya.

\section{Antibodies and reagents}

Detailed information on antibodies is summarized in Supplemental Table S4. Hexacosanoic acid (C26:0), propidium iodide (PI) and tunicamycin (TM) were purchased from Sigma (St. Louis, MO). The PERK inhibitor GSK2606414 was purchased from Calbiochem.

\section{Immunofluorescence and immunohistochemistry}

For all experiments, spinal cords were embedded in paraffin, and serial sections ( $4 \mu \mathrm{m}$ thick) were cut in a transversal or longitudinal ( $1 \mathrm{~cm}$ long) plane after perfusion with $4 \%$ paraformaldehyde.

For immunofluorescence studies, spinal cords from 3and 12-month-old wild type and $A b c d 1^{-}$mice were incubated with PDI, GRP78 or GFAP antibodies. Then, confocal images were acquired using a Leica TCS SL laser scanning confocal spectral microscope (Leica Microsystems Heidelberg $\mathrm{GmbH}$, Mannheim, Germany), and images were analysed with ImageJ $1.39 \mathrm{u}$ (NIH, USA). Details of the immunofluorescence processing can be found in the supplemental experimental procedures.

Immunohistological (IHC) studies performed in WT, $A b c d 1^{-} / A b c d 2^{-/-}$and $A b c d 1^{-} / A b c d 2^{-/-}$mice treated with TUDCA were carried out with the avidin-biotin peroxidase method, as reported earlier [65].

The results were expressed as the mean $\pm \mathrm{SD}$.

\section{Reverse transcription (RT)-PCR analysis}

Quantitative RT-PCR analysis was performed as previously described [65]. The expression of the genes of interest was analysed by Q-PCR using TaqMan ${ }^{\circledR}$ Gene Expression Assays (Applied Biosystems). Relative quantitation was carried out using the 'Delta-Delta $\mathrm{Ct}$ ' $(\Delta \Delta \mathrm{Ct})$ method with RPL0 as the endogenous control. Transcript quantification was performed in duplicate for each sample [65].

\section{Cell culture and treatment}

Primary human fibroblasts were cultured in DMEM (containing $10 \%$ foetal bovine serum, $100 \mathrm{U} / \mathrm{mL}$ penicillin and $100 \mu \mathrm{g}$ streptomycin) at $37{ }^{\circ} \mathrm{C}$ in humidified $5 \% \mathrm{CO}_{2} / 95 \%$ air. Unless otherwise stated, experiments were carried out with cells at $80 \%$ confluence. Cells were pretreated $1 \mathrm{~h}$ with $120 \mathrm{nM}$ of PERK inhibitor GSK2606414 and then exposed to $2 \mu \mathrm{g} / \mathrm{mL}$ of tunicamycin (TM) for $48 \mathrm{~h}$ or $72 \mathrm{~h}$. Cell lines were used on passages 12-18.

For viability experiments, cells were incubated with $10 \mu \mathrm{g} / \mathrm{mL}$ propidium iodide for $10 \mathrm{~min}$ in the dark after treatment and then analysed with an Agilent 2100 Bioanalyzer (Gallios Beckman Coulter). The apoptotic cells were quantified with FlowJo software.

\section{Statistical analysis}

Statistical significance was assessed using the Student's $t$ test whenever two groups were compared. When analysing multiple groups, we used a one-way ANOVA and Tukey's post hoc test to determine statistical significance. Data are presented as the mean $\pm \mathrm{SD}(* P<0.05 ; * * P<0.01$; $* * * P<0.001)$.

\section{Supplemental experimental procedures}

Details of XBPI mRNA processing, immunoblots and nuclear fractionation experiments can be found in the supplemental experimental procedures.

\section{Results}

In this work, we analysed three different types of samples associated with X-ALD, including necropsy samples of brain white matter from patients with cerebral inflammatory disease (CCALD and cAMN), patient fibroblasts, and spinal cords from the X-ALD mouse model $\left(A b c d 1^{-}\right.$and $A b c d 1^{-} / A b c d 2^{-1-}$ mice). We examined the following markers of UPR activation: (i) the induction of the ER stress sensors PERK, ATF6 and IRE1 and (ii) the expression of the UPR responsive proteins ATF4, CHOP, GADD34, GRP78, GRP94 and PDI.

\section{The UPR is induced in the brain samples of patients with X-ALD}

Analysis of PERK phosphorylation and the P-eIF2 $\alpha /$ eIF2 $\alpha$ ratio showed an increase in PERK and eIF2 $\alpha$ phosphorylation levels in the affected areas of CCALD and cAMN patients (Fig. 1a, b). In addition, we found a significant up-regulation of ATF4 levels in the affected areas of CCALD and cAMN patients, which correlated with an induction of CHOP in the affected areas of both phenotypes (Fig. 1a, b). However, for the same samples, no change was detected in the levels of GADD34, another target gene of ATF4 (Fig. 1a, b).

We assessed ATF6 activation by measuring the liberation of its cleaved fragment. We showed lower cleavedATF6 levels in the affected areas of the CCALD and cAMN brains than in the normal-appearing areas and 
matched healthy individuals (Fig. 1a, b). Finally, immunoblot experiments showed that the levels of the IRE1 transducer were decreased in the affected zones of CCALD and cAMN brains (Fig. 1a, b). In addition, both unspliced and spliced XBPI mRNA levels were similar in control, non-affected and affected white matter, indicating that the IRE1 pathway was not overactivated in X-ALD patients (Fig. 1c, d).

In affected white matter samples of CCALD and cAMN patients, ATF6 mRNA and protein shared the same pattern of repression, which was correlated with decreased expression of GRP78, GRP94, EDEM2 and HERPUD1 mRNA levels, (Supplemental Fig. S1a, b). However, protein expression of GRP78, GRP94 and PDI was induced in the same samples (Fig. 1e, f). That may be due to a lack of degradation of GRP78 and GRP94, which may undergo posttranslational modifications such as S-nitrosylation, as shown for PDI and GRP78 [23, 25, 26]. Under normal conditions, these proteins would be degraded by the proteasome or autophagy routes, which may not occur in X-ALD as both systems are malfunctioning, as we have previously reported $[8,9]$.

In conclusion, the PERK pathway but not the IRE1 or ATF6 pathways are activated in the affected white matter of CCALD and cAMN patients. In the spinal cords from cAMN patients, we obtained similar results regarding PERK activation and for the ATF6 pathway which was not modified (Supplemental Fig. S2a, b).

\section{Pathway-specific regulation of the UPR in $\mathrm{Abcd1}^{-}$mice}

We measured the activation of ER sensors in the spinal cords from $A b c d 1^{-}$mice early in adulthood, at three months and at 12 months of age, well prior to disease onset, which occurs at 20 months. PERK phosphorylation and the P-eIF2 $\alpha /$ eIF $2 \alpha$ ratio were increased in X-ALD mice at 12 months but not at three months of age. This PERK pathway activation was confirmed by enhanced ATF4 and CHOP levels in $A b c d l^{-}$spinal cord tissue starting at 12 months. Finally, in agreement with the observations in the brain samples, we could not detect differences in the GADD34 levels at either three or 12 months of age (Fig. 2a). In contrast, here we detected increased levels of cleaved-ATF6 at three, and to a smaller extent, 12 months (Fig. 2a). To confirm the activation of the ATF6 pathway, we analysed the translocation of cleaved-ATF6 into the nucleus and found that cleaved-ATF6 was increased in $A b c d 1^{-}$mouse spinal cord tissue at three, and to a lesser extent, 12 months (Fig. 2b). Since ATF6 also activates genes involved in the ERAD, we measured the expression of Edem 2 and Herpudl mRNA at 12 months of age. Indeed, expression of these ERAD genes appeared to be induced (Fig. 2c). The IRE1 transducer was not activated at three or 12 months, as demonstrated by the lack of spliced XBP1 (mRNA and protein) in $A b c d 1^{-}$mouse spinal cord tissue (Supplemental Fig. S3a, b).

Taken together, these data indicate that the ER stress response is an early phenomenon in the X-ALD mouse model, with a strong activation of the ATF6 pathway at three months of age and a later activation of the PERK and ATF6 but not IRE1 pathways.

\section{Time-dependent expression of chaperones and PDI in motor neurons and astrocytes of $\mathrm{Abcd1}^{-}$mice}

The expression of the mRNA and protein of the chaperones GRP78, GRP94 and PDI was up-regulated in the total extracts from the spinal cord of 3-month-old $A b c d 1^{-}$ mice. At 12 months of age, the increase in PDI persisted (protein and mRNA), whereas GRP78 and GRP94 protein levels were down-regulated in the mutant mice compared with WT littermates (Fig. 2d). Moreover, Grp78 and Grp94 mRNA levels were not altered and increased, respectively, at this age in $A b c d 1^{-}$mice (Supplemental Fig. S3c). To gain insight into the cell-type specificity of the observed response, we performed immunofluorescence in spinal cord sections. Prominent GRP78 and PDI immunostaining was observed in motor neurons of the $A b c d 1^{-}$mouse spinal cord at three months compared with that in the WT mice (Fig. 3a), which is consistent with the results obtained by western blot (Fig. 2d). We also detected an up-regulation of GRP78 expression but not an increase in PDI staining in astrocytes of $A b c d 1^{-}$mice at three months of age (Fig. 3b). At 12 months of age, we observed results similar to those obtained by western blot (i.e. decreased expression of GRP78 in both motor neurons and astrocytes and an induction of PDI staining in motor neurons from $A b c d 1^{-}$mice) (Fig. 3a, b).

\section{UPR induction in fibroblasts from X-ALD patients}

The fibroblasts of X-ALD patients are a good surrogate cell model for dissecting disease mechanisms, as they recapitulate the main X-ALD hallmarks: (i) accumulation of VLCFA [101], (ii) higher production of free radicals of mitochondrial origin [58], (iii) loss of energetic homeostasis [34], and (iv) altered proteostasis [55, 56]. Using this cell system, we sought to determine whether an induction of ER stress was detected in fibroblasts from X-ALD patients.

At baseline, we found increases in the levels of cleavedATF6, ATF4, CHOP, and PERK and eIF2 $\alpha$ phosphorylation, thereby mimicking the pattern of ER stress sensor activation in the spinal cords of 12-month-old $A b c d 1^{-}$mice (Fig. 4a). Moreover, we detected an increase in the protein levels of GRP94 and GRP78 in X-ALD fibroblasts compared to that in the control cells at baseline (Fig. 4a). 
a

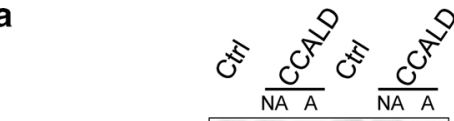
ATF6
cleaved-ATF6

P-PERK $\rightarrow-\square$

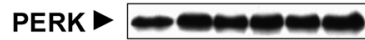

P-elF2 $\alpha$

elF2 $\alpha \longrightarrow--\ldots$

ATF4 $>$.

CHOP $\square \square$

GADD34 — —

IRE1 $--\cdots-\cdots$

$\gamma$-Tub $\approx=-\infty-\infty$

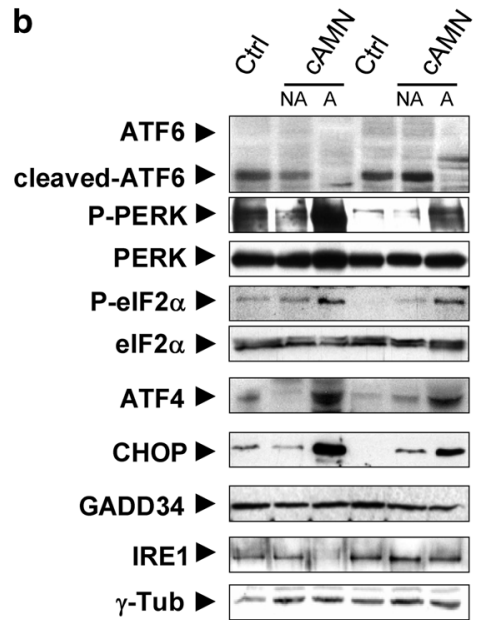

C

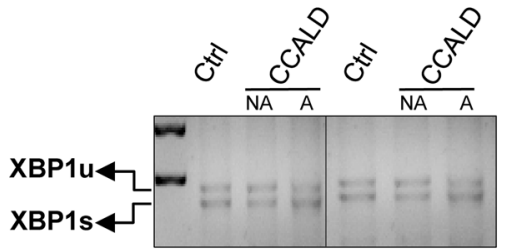

e
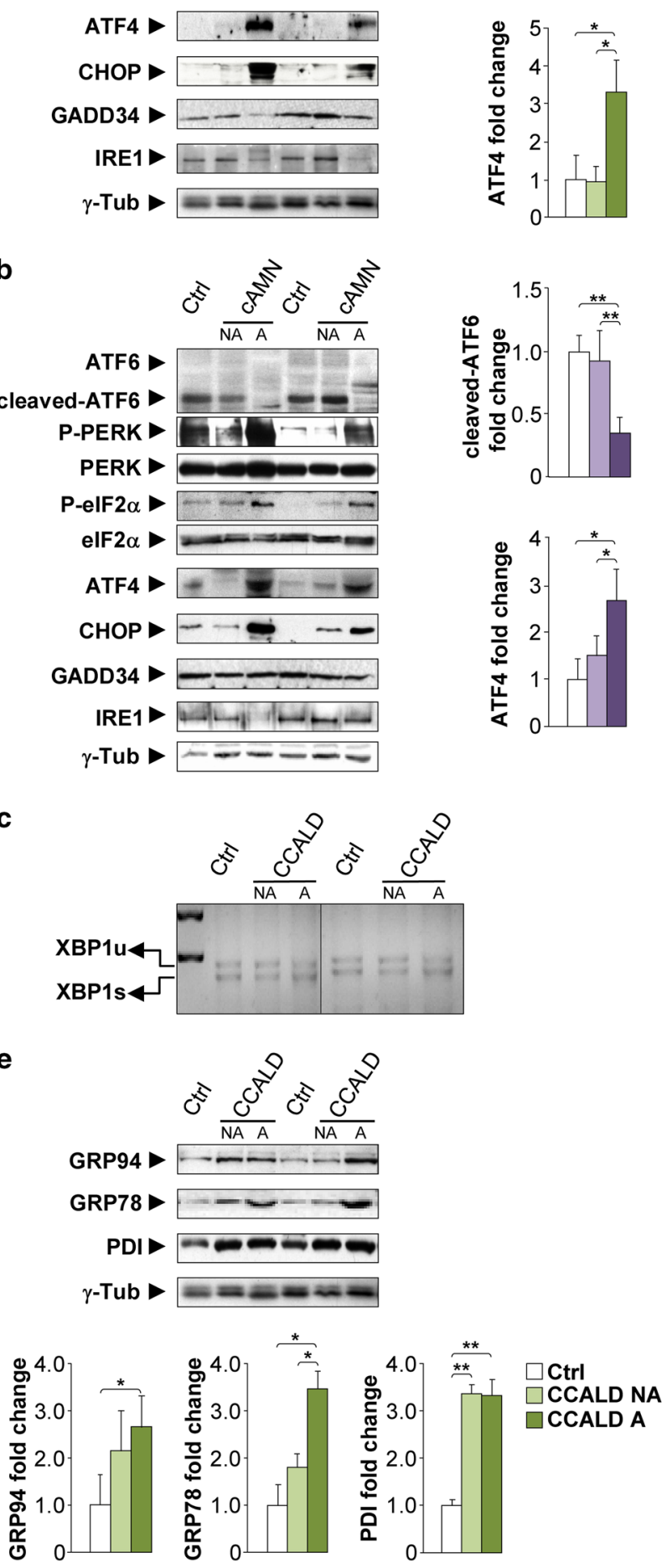
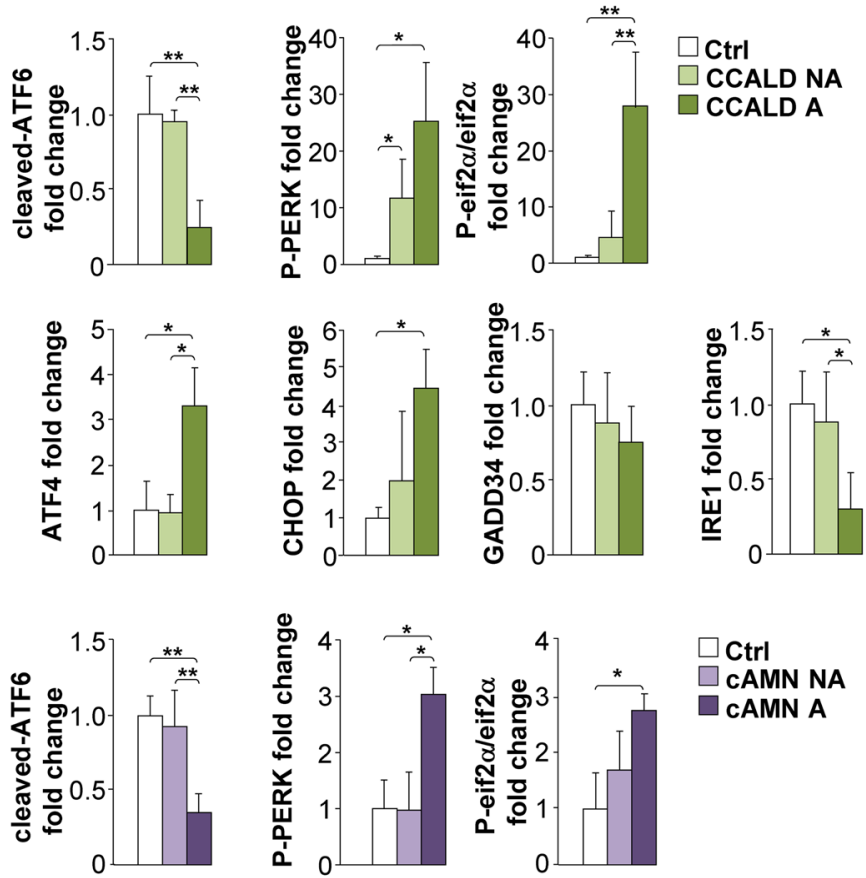

$$
\begin{aligned}
& \square \text { Ctrl } \\
& \square \text { CAMN NA } \\
& \square \text { CAMN A }
\end{aligned}
$$
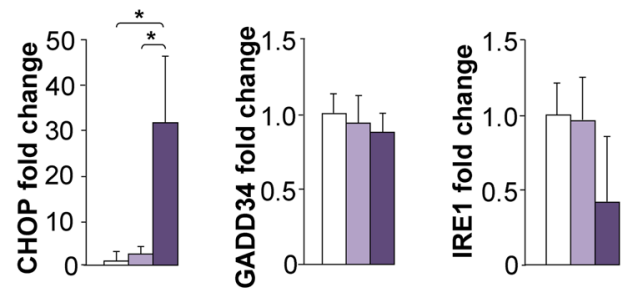

d

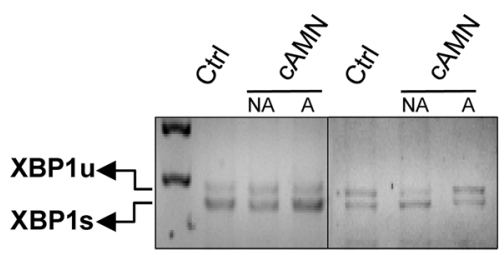

f
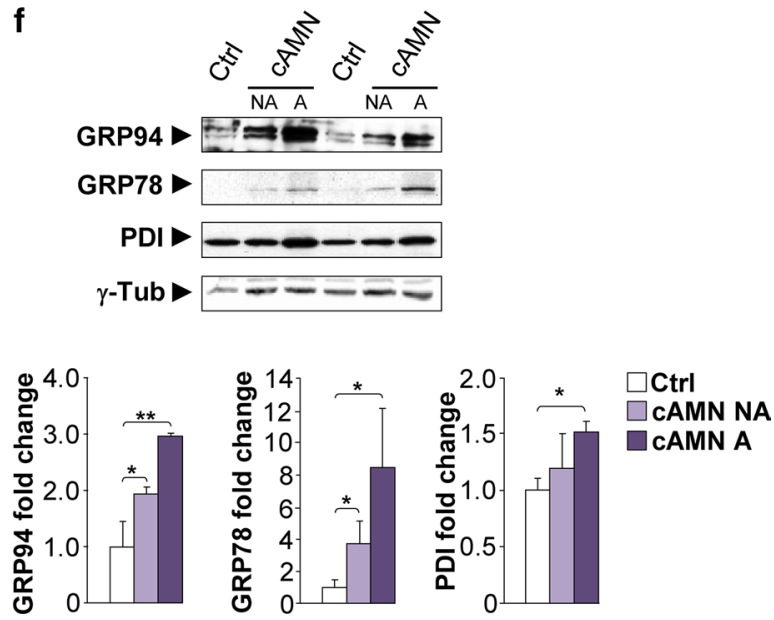
4Fig. 1 a, b Induction of the UPR in CCALD and CAMN patient brains. Representative immunoblots for full-length ATF6, cleavedATF6, total PERK, phosphorylated PERK (P-PERK), eIF2 $\alpha$, phosphorylated-eIF2 $\alpha$ (P-eIF2 $\alpha$ ), ATF4, CHOP, GADD34 and IRE1 levels in total lysates from controls (Ctrl) and in normal-appearing (NA) and affected (A) white matter from CCALD (a) and cAMN patients (b). XBP1 mRNA splicing analysis by RT-PCR in Ctrl samples and NA and A white matter from CCALD (c) and cAMN patients (d). Unspliced- and spliced-XBP1 mRNA corresponds to $X B P 1 u$ mRNA and XBP1s mRNA, respectively. Representative immunoblots for GRP78, GRP94, and PDI levels in Ctrl samples and NA and A white matter from CCALD (e) and cAMN patients (f). Protein levels were normalized relative to $\gamma$-tubulin $(\gamma$-Tub). The histograms on the right $(\mathbf{a}, \mathbf{b})$ and below $(\mathbf{e}, \mathbf{f})$ show the ratio and the protein levels relative to control. All values are expressed as the mean $\pm \mathrm{SD}(n=5$ by genotype and condition in $\mathbf{a}-\mathbf{f}$; $* P<0.05$ and $* * P<0.01$, one-way ANOVA followed by Tukey's HSD post hoc test for $\mathbf{a}, \mathbf{b}, \mathbf{e}$ and $\mathbf{f}$ )

\section{Inhibition of PERK disrupts the UPR and renders the X-ALD fibroblasts more susceptible to ER stress}

To determine whether UPR activation was protective or detrimental in the X-ALD fibroblasts, we targeted the UPR machinery using tunicamycin (TM), which blocks $\mathrm{N}$-linked glycosylation, inducing protein misfolding and the UPR. After a 48-h incubation with $2 \mu \mathrm{g} / \mathrm{mL} \mathrm{TM}$, we detected a slight but significant $10 \%$ increase in cell death in the X-ALD fibroblasts compared to that in the controls (Supplemental Fig. S4). In these conditions, we found that the PERK pathway was induced in the X-ALD fibroblasts as they presented significantly higher ATF4, CHOP and GADD34 protein levels than baseline (Fig. 4a). Of note, the mRNA levels of Edem2 and Herpudl were strongly increased in X-ALD fibroblasts upon tunicamycin treatment, suggesting that the ERAD machinery was more reactive to TM-induced stress (Fig. 4b). In the control fibroblasts, we found a similar pattern but a less intense response.

Since the PERK pathway appeared to be a pivotal player in the TM-induced UPR activation, we assessed the effects of the specific PERK inhibitor GSK2606414 on the observed UPR-induced cell death. We found that the PERK inhibitor induced a more premature cell death in X-ALD than control fibroblasts following $48 \mathrm{~h}$ with $2 \mu \mathrm{g} /$ $\mathrm{mL}$ TM (25\% for X-ALD and $12 \%$ for controls), while GSK2606414 alone did not have any effect on cell death (Supplemental Fig. S4). At basal levels, GSK2606414 significantly decreased PERK phosphorylation and CHOP levels in X-ALD fibroblasts in comparison with control fibroblasts.

Following $2 \mu \mathrm{g} / \mathrm{mL}$ TM plus $120 \mathrm{nM}$ GSK2606414 treatment for $48 \mathrm{~h}$, we found a significant decrease in P-PERK, P-eIF2 $\alpha$, ATF4 and CHOP levels in X-ALD fibroblasts. In contrast to control fibroblasts, the only significant decrease was in the expression of CHOP (Fig. 4a). Interestingly, the GRP94 and GRP78 protein levels and Edem 2 and Herpudl mRNA levels were also substantially reduced with PERK inhibition in the $\mathrm{X}$-ALD fibroblasts following $48 \mathrm{~h} \mathrm{TM}$ treatment (Fig. 4a, b). These data suggest that PERK is a major participant in the transcriptional control of ATF6 and many UPR target genes in response to tunicamycin treatment in $\mathrm{X}$-ALD fibroblasts, including those involved in protein folding, such as GRP94 or GRP78 and ERAD factors such as EDEM2 or HERPUD1.

Altogether, these results suggest that PERK inhibition blocks the induction of eIF $2 \alpha$ phosphorylation and the translational control arm of the UPR, which leads to disruption of homeostasis and increased apoptosis during ER stress induced in X-ALD fibroblasts. Theses results were in accordance with the study by Teske et al. [88], showing that the PERK pathway facilitates both the synthesis of ATF6 and trafficking of ATF6 from the ER to the Golgi for intramembrane proteolysis and activation of ATF6. As a consequence, PERK loss of function could significantly reduce both the translational and transcriptional phases of the UPR, leading to reduced protein chaperone expression and enhanced apoptosis.

\section{Antioxidant treatment prevents ER stress and UPR induction in $\mathrm{Abcdl}^{-}$mice}

We previously demonstrated that VLCFA excess produces free radicals, and as a consequence, early oxidative damage is present in the spinal cords of $A b c d 1^{-}$mice [29]. A combination of vitamin E, $\alpha$-lipoic acid and NAC efficiently reduced ROS production in vitro, reversed oxidative damage to proteins and DNA in spinal cords, and arrested axonal degeneration and disability in X-ALD mice [57]. To determine whether UPR is regulated by redox imbalance in vivo, we treated the presymptomatic $A b c d 1^{-}$mice at eight months of age with the above-mentioned combination of antioxidants for four months. The antioxidant treatment normalized the levels of cleaved-ATF6, phosphorylated PERK, phosphorylated eIF2 $\alpha$, ATF4 and CHOP (Fig. 5a), GRP94, GRP78 and PDI (Fig. 5b) and Edem2 mRNA (Fig. 5c) in $A b c d 1^{-}$mice. These data demonstrate that oxidative stress is an upstream promoter of UPR activation in $\mathrm{X}$-ALD mice.

\section{The bile acid TUDCA reduces the UPR activation in $\mathrm{Abcd1}^{-}$mice}

Chemical chaperones have previously been shown to be effective at reducing ER stress [70, 72]. TUDCA, which is a well-known member of this chemical chaperone family, 

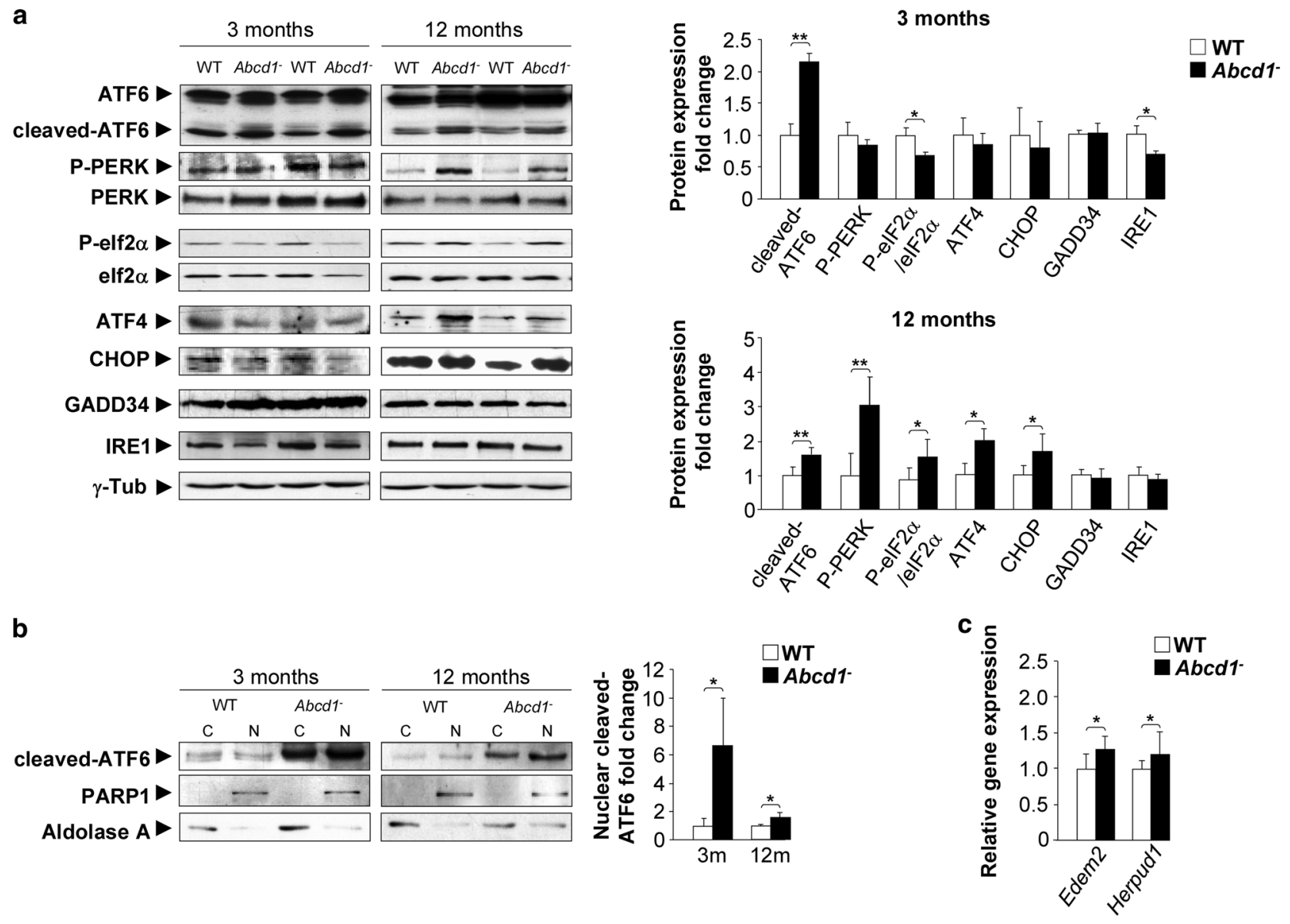

d
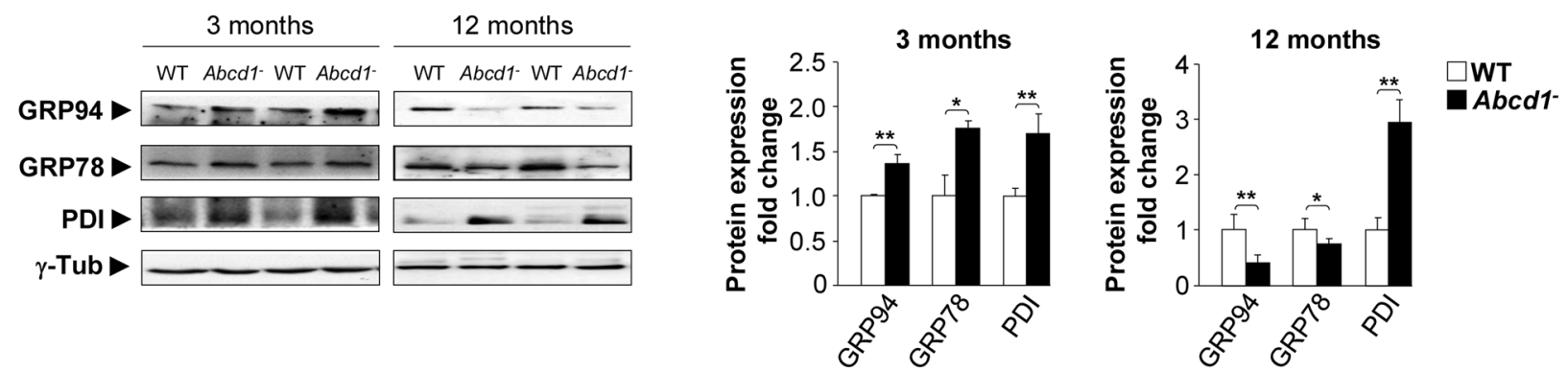

Fig. 2 UPR induction in the X-ALD mouse model. a Representative immunoblots of ER stress sensors full-length ATF6, cleaved-ATF6, total PERK, P-PERK, eIF2 $\alpha$, P-eIF2 $\alpha$, ATF4, CHOP, GADD34 and IRE1 in the spinal cord tissue of $A b c d 1^{-}$mice and age-matched wild type (WT) mice at 3 and 12 months of age. The histograms on the right show the cleaved-ATF6, P-PERK, ATF4, CHOP, GADD34 and IRE1 levels normalized relative to $\gamma$-Tub and the P-PERK/PERK and the P-eIF2 $\alpha /$ eIF $2 \alpha$ ratios relative to their respective WT values. $\mathbf{b}$ The nuclear localization of cleaved-ATF6 in WT and $\mathrm{Abcd1}^{-}$mice at 3 and 12 months of age. PARP1 was used as the control for the nuclear fraction $(\mathrm{N})$ and aldolase A was used for the cytoplasmic fraction $(\mathrm{C})$.

is a unique bile acid that acts as a potent anti-apoptotic agent. Previous studies have shown its involvement as a neuroprotective agent in neurodegenerative diseases where the UPR is considered to be a trigger of pathogenic

The histograms on the right show the cleaved-ATF6 levels relative to WT values in the nuclear fractions. c Real-time RT-PCR analyses of Edem 2 and Herpud1 mRNA at 12 months in $A b c d 1^{-}$mouse spinal cords. d Immunoblots of GRP94 and GRP78 chaperones and PDI in the spinal cord tissue of $A b c d 1^{-}$mice and age-matched wild type (WT) mice 3 and 12 months of age. The histograms on the right show the GRP94 and GRP78 chaperones and PDI levels normalized to WT mice and normalized relative to the $\gamma$-Tub. Values are expressed as the mean $\pm \mathrm{SD}$ ( $n=10$ samples per genotype and condition in $\mathbf{a}$, $\mathbf{c}$ and $\mathbf{d} ; n=6$ samples per genotype and condition in $\mathbf{b} ; * P<0.05$, $* * P<0.01$ and $* * * P<0.001$, Student's $t$ test)

mechanisms, such as AD [17], PD [10] and ALS [106]. Accordingly, $A b c d 1^{-}$mice were fed a diet that was supplemented with $0.4 \%(\mathrm{wt} / \mathrm{wt})$ TUDCA for three months starting at nine months of age. Then, we assessed the protein 
a
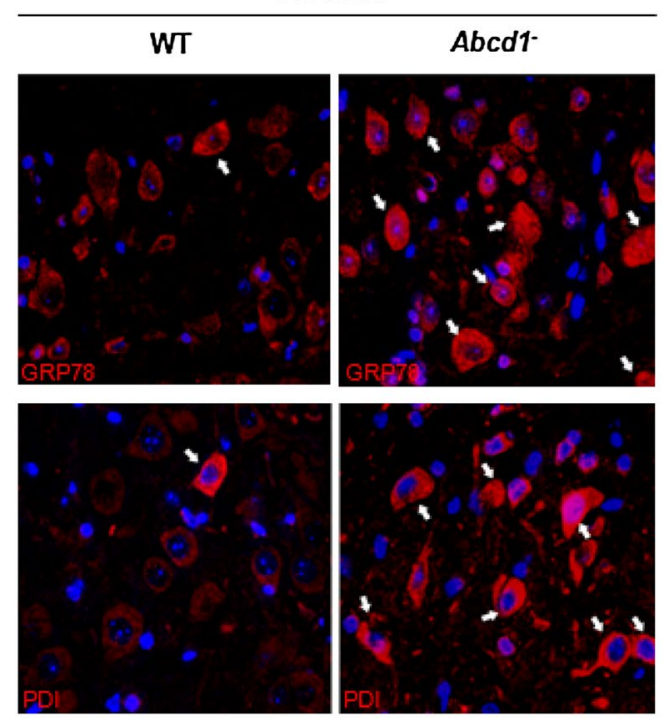

b

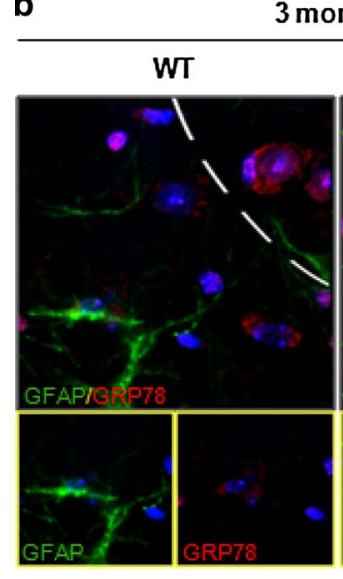

3 months
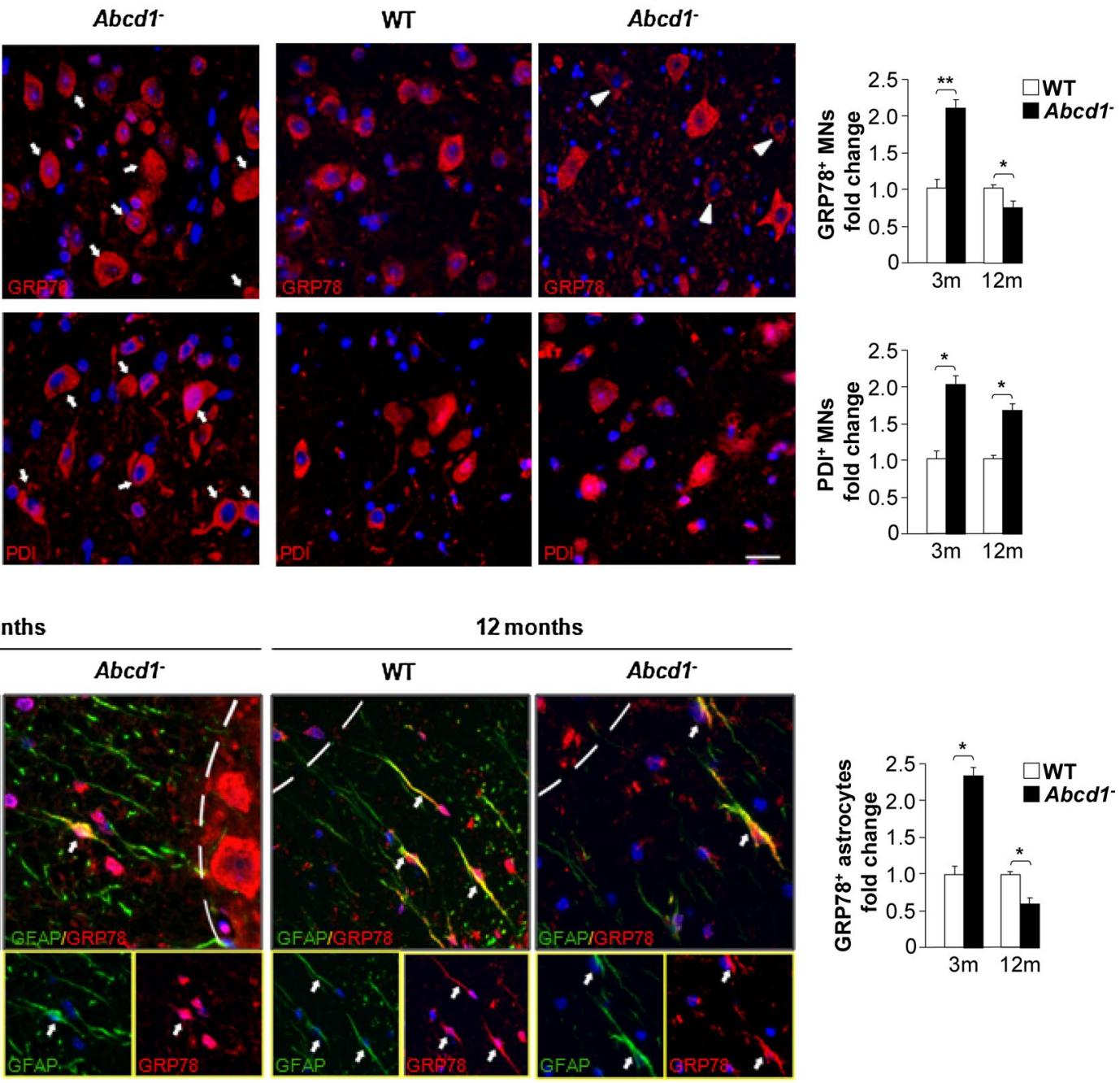
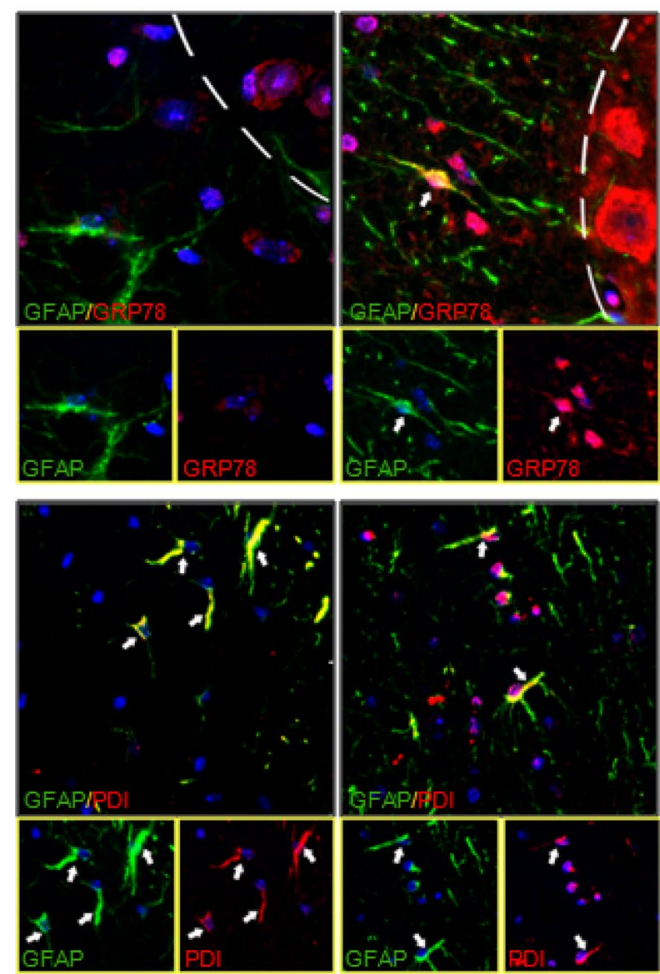
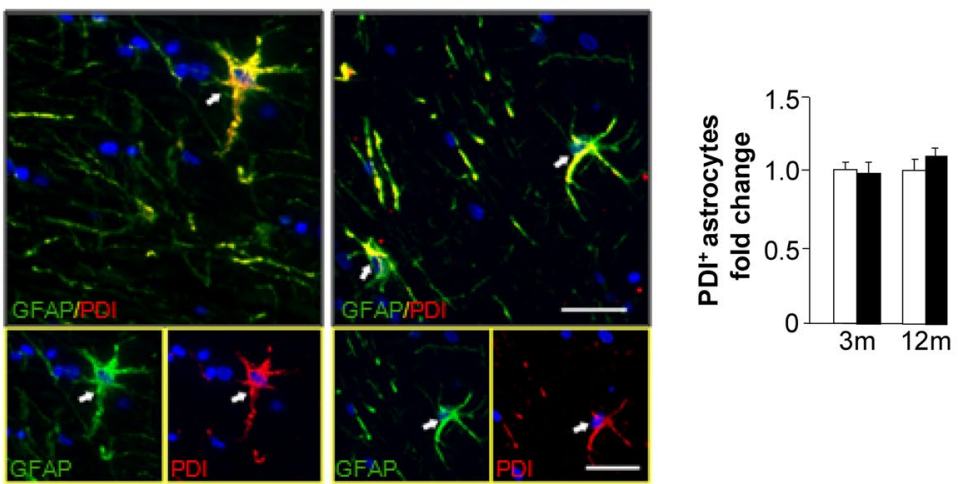

Fig. 3 The UPR is primarily induced in motor neurons and astrocytes during X-ALD pathogenesis. a Immunofluorescence of GRP78 and PDI in spinal cord sections of WT and $A b c d 1^{-}$mice 3 and 12 months of age. Arrows indicate high amounts of GRP78 and PDI in motor neurons; Arrowheads denote motor neurons with low amounts of GRP78. Nuclei were counterstained with DAPI. Scale bar $50 \mu \mathrm{m}$. b Dual immunolabelling of astrocytes (GFAP; green) with GRP78 or PDI (red) in spinal cord sections of WT and $A b c d 1^{-}$mice 3 and 12 months of age. Positive cells are labelled with arrows, and the dashed-line stands for the limit between the grey- and white matter. Nuclei were counterstained with DAPI. The small panel below shows some double-positive astrocytes. Scale bar $25 \mu \mathrm{m}$. The histogram on the right represents the quantification of GRP78 and PDI fluorescence intensity normalized to WT mice in motor neurons (MNs) (a) and astrocytes (b). Values are expressed as the mean $\pm \mathrm{SD}$ $(n=4$ samples per genotype and condition in $\mathbf{a}$ and $\mathbf{b} ; * P<0.05$, $* * P<0.01$ and $* * * P<0.001$, Student's $t$ test) 
Fig. 4 Inhibition of PERK disrupts the UPR and renders the X-ALD fibroblasts more susceptible to ER stress. a Representative immunoblots for cleaved-ATF6, total PERK, P-PERK, total eIF $2 \alpha$, P-eIF $2 \alpha$, ATF4, CHOP, GADD34, GRP78, GRP94 and PDI levels in control (Ctrl) and $\mathrm{X}$-ALD human fibroblasts pretreated with or without the PERK inhibitor GSK2606414 (GSK, $120 \mathrm{nM}$ ) for $1 \mathrm{~h}$ and then exposed to tunicamycin (TM, $2 \mu \mathrm{g} / \mathrm{mL}$ ) for $48 \mathrm{~h}$. Protein levels were normalized relative to $\gamma$-tubulin $(\gamma$-Tub). The histograms below show the cleaved-ATF6, P-PERK, P-eIF2 $\alpha$, ATF4, CHOP, GADD34, GRP94, GRP78 and PDI levels normalized relative to $\gamma$-Tub and the P-PERK/ PERK and P-eIF $2 \alpha /$ IF $2 \alpha$ ratios relative to their respective WT values. b Real-time RT-PCR analyses of Edem 2 and Herpudl mRNA levels in Ctrl and X-ALD human fibroblasts pretreated with or without the PERK inhibitor GSK2606414 (GSK; $120 \mathrm{nM}$ ) for $1 \mathrm{~h}$ and then exposed to tunicamycin (TM, $2 \mu \mathrm{g} / \mathrm{mL}$ ) for $48 \mathrm{~h}$. All values are expressed as the mean \pm SD ( $n=4$ by genotype and condition; $* P<0.05$ and $* * P<0.01$, one-way ANOVA followed by Tukey's HSD post hoc test) a

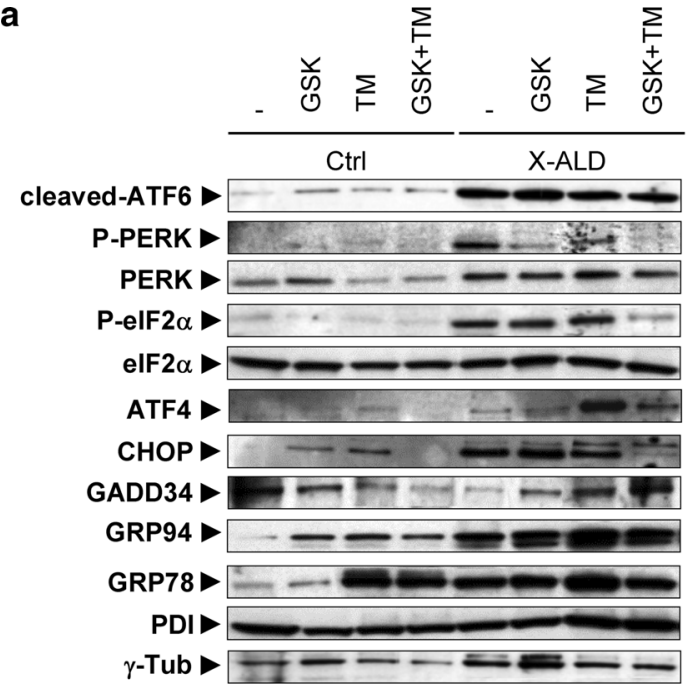

$\square$ Ctrl

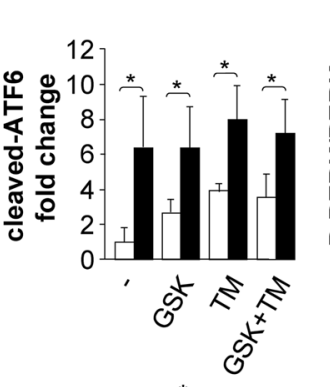

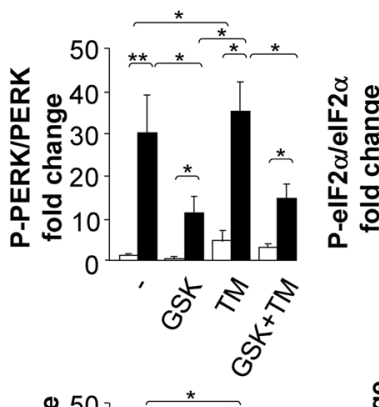
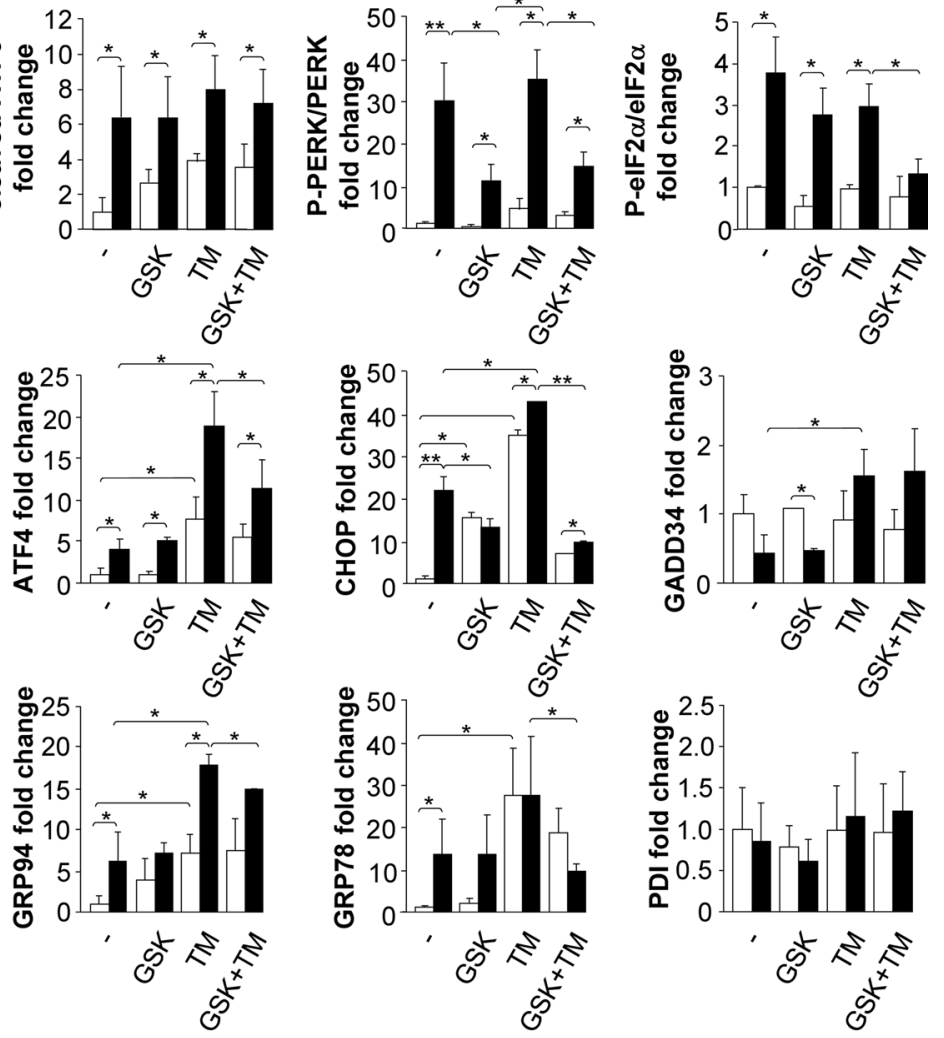

b
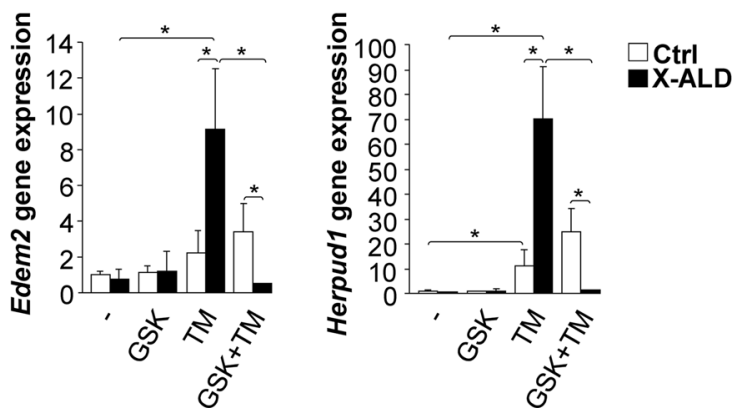

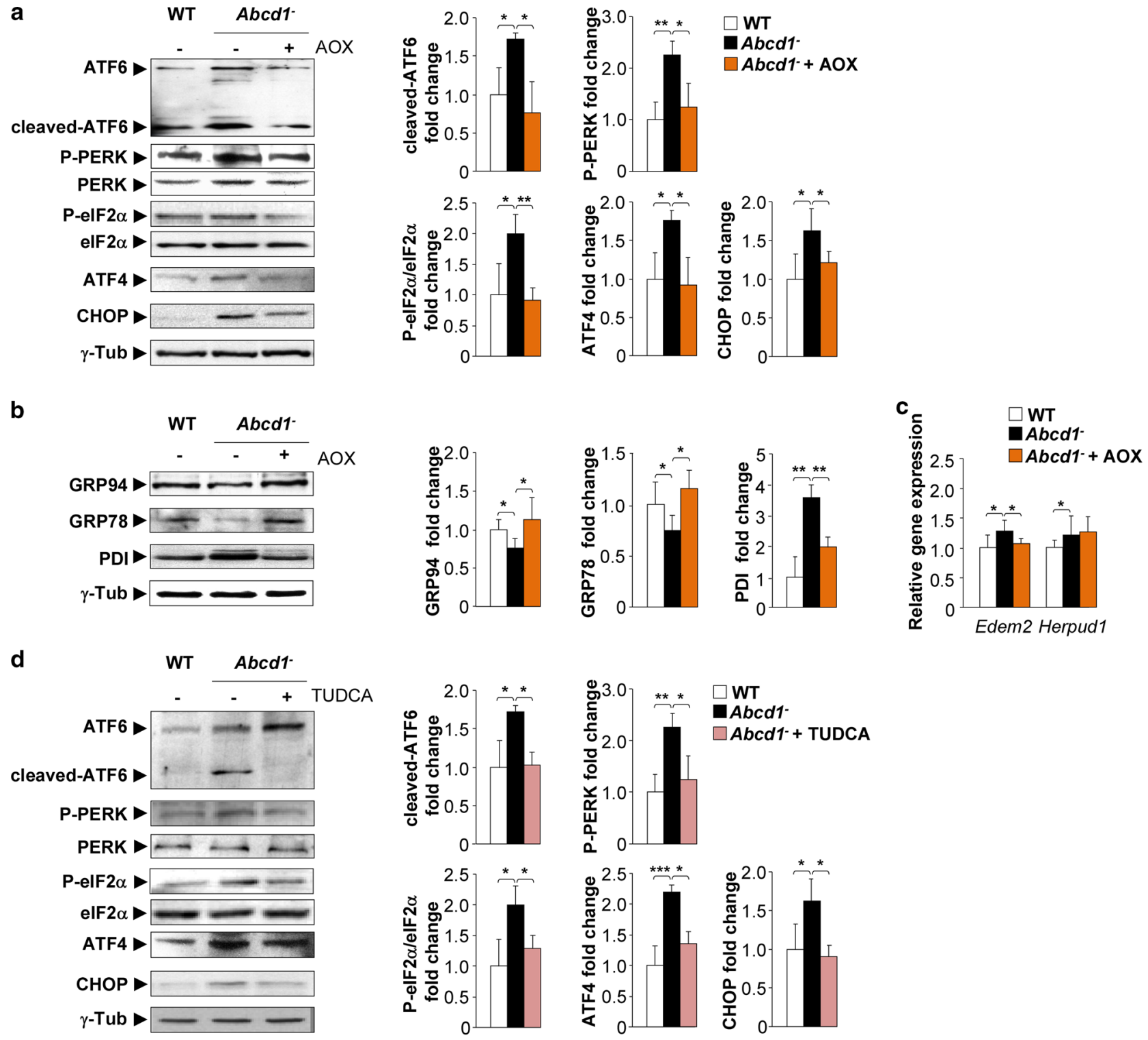

e
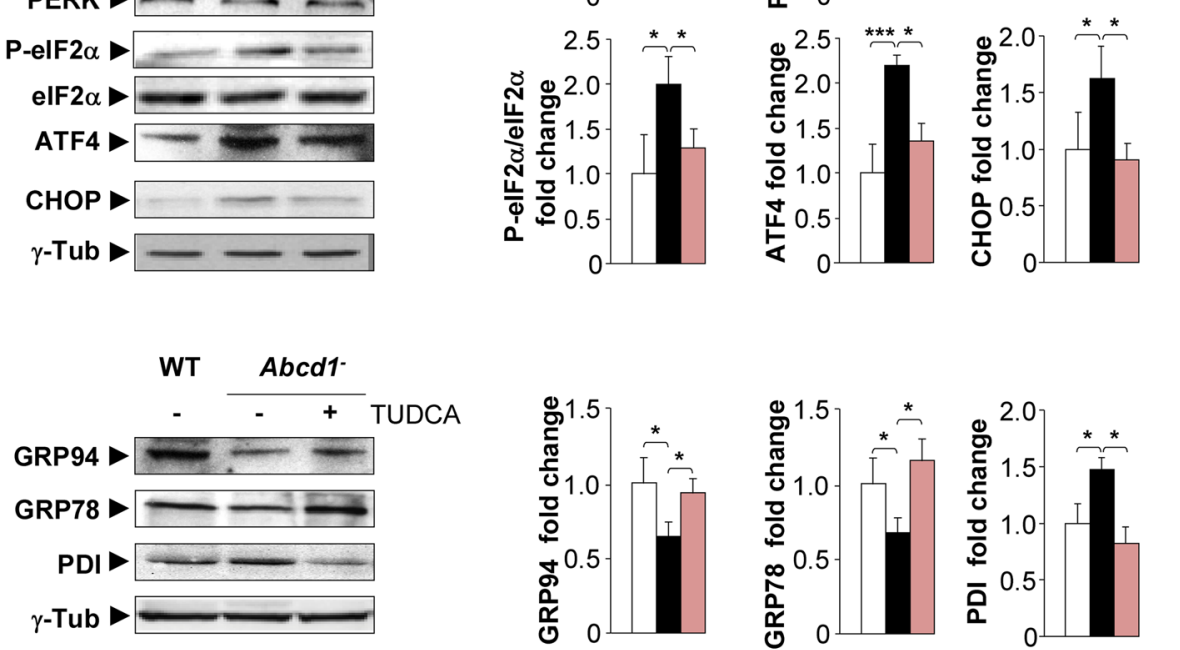

Fig. 5 Antioxidant and TUDCA treatments prevent UPR activation in the X-ALD mouse model. a, d Representative immunoblots for full-length ATF6, cleaved-ATF6, total PERK, P-PERK, total eIF $2 \alpha$, P-eIF2 $\alpha$, ATF4 and CHOP in the spinal cord tissue of WT mice (WT), $A b c d 1^{-}$mice $\left(A b c d 1^{-}\right)$and a antioxidant-treated $\left(A b c d 1^{-}+\mathrm{AOX}\right)$ or d TUDCA-treated $\left(A b c d 1^{-}+\right.$TUDCA) $A b c d 1^{-}$ mice at 12 months of age. GRP78, GRP94 and PDI levels were analysed in the spinal cords of WT, $A b c d 1^{-}$and $A b c d 1^{-}+\mathrm{AOX}$ mice (b) and $A b c d 1^{-}+$TUDCA mice (e) at 12 months of age. Real-time

RT-PCR analyses of Edem 2 and Herpudl in the spinal cord tissue of WT mice (WT), $A b c d 1^{-}$mice $\left(A b c d 1^{-}\right)$and $\mathbf{c}$ antioxidanttreated $\left(A b c d 1^{-}+\mathrm{AOX}\right)$ or $\mathbf{f}$ TUDCA-treated $\left(A b c d 1^{-}+\right.$TUDCA) $A b c d 1^{-}$mice at 12 months of age. In $(\mathbf{a}, \mathbf{b}$ and $\mathbf{d}, \mathbf{e})$, the histograms on the right show normalized UPR marker levels relative to those in untreated WT mice. All values are expressed as the mean $\pm \mathrm{SD}(n=10$ by genotype and condition in $\mathbf{a}-\mathbf{d}$; $* * P<0.01$ and $* * * P<0.001$, one-way ANOVA followed by Tukey's HSD post hoc test) 
levels of several UPR responsive genes and ER stress sensors that were previously identified as being dysregulated in the $A b c d 1^{-}$mice. The levels of cleaved-ATF6, P-PERK, P-eIF2 $\alpha /$ eIF2 $\alpha$, ATF4 and CHOP (Fig. 5d), the levels of GRP78 and GRP94 and PDI (Fig. 5e) and the mRNA levels of Edem 2 and Herpudl (Fig. 5f) were normalized in $A b c d 1^{-}$mice following exposure to TUDCA.

\section{TUDCA halts axonal degeneration and locomotor deficit progression in $\mathrm{Abcd1}^{-} / \mathrm{Abcd2}^{-/-}$mice}

We first observed that the UPR was also activated in $A b c d 1^{-} / A b c d 2^{-1-}$ mice, following a similar pattern than in $A b c d 1^{-}$mice (Supplemental Fig. S5). To assess the preclinical potential of the treatment, we treated with TUDCA cohorts of $A b c d 1^{-} / A b c d 2^{-/-}$right at disease onset, starting at 13 months of age until 17 months of age. As observed in $A b c d 1^{-}$mice (Fig. 5d, e), TUDCA is able to inhibit UPR activation in this model (Supplemental Fig. S5). We then evaluated axonal degeneration by semi-quantifying the accumulation of the axonal damage markers synaptophysin and APP, the scattered myelin debris with Sudan black staining, the number of reactive microglia and reactive astrocytes and its activated-state morphology. Therefore, we observed that these measures were strikingly reduced to control levels following TUDCA treatment (Fig. 6a-q; Supplemental Table S5).

We next evaluated locomotor deficits in $\mathrm{Abcdl}^{-} /$ $A b c d 2^{-1-}$ mice using clasping [19], treadmill and bar cross experiments [55, 57, 64, 65] after TUDCA treatment. In the clasping test, the best performance score of each animal was used for statistical analysis. Compared with WT mice, $A b c d 1^{-} / A b c d 2^{-/-}$mice presented a lower score, thereby demonstrating a locomotor deficit (Fig. 6r). Remarkably, TUDCA treatment normalized this score. In the treadmill experiment, the double-knockout mice required longer shock times and more frequent shocks to run at belt speed than the WT mice, as described earlier [55, 57, 64, 65]. After four months of treatment, this ratio was indistinguishable from the WT ratio (Fig. 6s). Finally, in the bar cross experiments, double-knockout mutants often failed to maintain their balance and displayed a greater tendency of the hind limbs to slip off of the bar and longer latencies to reaching the platform at the opposite end of the bar [55, 57, 64, 65]. The number of slips and the time necessary to cross the bar were also normalized following TUDCA treatment (Fig. 6t). Overall, these data show that TUDCA treatment arrested the progression of the locomotor deficits that occur in $A b c d 1^{-} / A b c d 2^{-/-}$ mice. Altogether, these results established a direct link between UPR activation, axonal degeneration and locomotor deficits in X-ALD.

\section{Discussion}

\section{An ER stress signature in peroxisomal disorders?}

Peroxisomes are oxidative organelles present in all tissues and organisms [83, 84, 102, 103] and are involved in several anabolic and catabolic reactions, including the following: the degradation of long- and very long-chain fatty acids (LCFA, VLCFA ( $\mathrm{C} \geq 22: 0)$, respectively) by $\alpha$ - or $\beta$-oxidation and hydrogen peroxide detoxification and the synthesis of bile acids, plasmalogens and essential polyunsaturated fatty acids. The loss or malfunction of peroxisomes is the cause of more than 20 fatal inherited conditions, which are classified into two main groups: (i) peroxisome biogenesis (PBD) and (ii) single peroxisomal enzyme deficiencies. Mutations in peroxisomal proteins that are essential for biogenesis and membrane protein import (called peroxins or PEX genes) invariably lead to PBD, with Zellweger syndrome as the most severe manifestation [3, 16, 83, 102, 103], (see also http://www.peroxisomedb.org).

Morphological abnormalities of the ER compartment have been described in peroxisomal mutants, including $P e x 5^{-/-}$[18] and $A b c d 2^{-I-}$ mice [27]. Interestingly, previous studies using $P e x 2^{-/-}$as a model of PBD [53, 54] or Acox $1^{-1-}$ as a model of single peroxisomal enzyme deficiency [45] have shown that ER stress is induced. Indeed, the ER stress response in the $P e x 2^{-/-}$liver revealed a predominant activation of specific UPR branches (i.e. the PERK-ATF4 pathway), with increased mRNA expression of CHOP in conjunction with a lack or paucity of IRE1 activation and the absence of subsequent XBP1 mRNA splicing [54]. The panoply of metabolic abnormalities in $P e x 2^{-/-}$liver tissue $[24,99]$ includes the perturbed flux of mevalonate, bile acids, plasmalogens, docosahexaenoic acid (DHA, C22:6w3) and VLCFA. Moreover, the same type of UPR activation (excluding IRE1) occurs in $A \operatorname{cox} 1^{-1-}$ liver tissue [45], in which the first enzyme of the peroxisomal $\beta$-oxidation, the so-called fatty acylCoA oxidase 1 , is inactive; thus, only the levels of DHA and VLCFA but not plasmalogens or bile acid are altered [2, 45, 48]. In $A b c d 1^{-}$mice with peroxisomal malfunctions that are largely related to defective import-induced excessive VLCFA, we observed a similar UPR pattern with predominant activation of the PERK-ATF4 pathway and a blunted IRE1/Xbp1 response at 12 months of age.

This finding is in contrast with the phenotype in models of other leukodystrophies, such as vanishing white matter disease and Pelizaeus-Merzbacher disease (PMD), in which all three branches of the UPR are activated [86, 92]. The same observations occur in models of ALS [51], AD [52], tauopathies [39, 52] and synucleinopathies [12, 13]. 
Fig. 6 a-q TUDCA halts axonal degeneration and locomotor disability in $\mathrm{Abcd1}^{-}$, $A b c d 2^{-l-}$ mice. Immunohistological analysis of axonal pathologies performed on WT, $A b c d 1^{-} / A b c d 2^{-/-}$and TUDCAtreated $A b c d 1^{-} / A b c d 2^{-/-}$mice $\left(A b c d 1^{-} / A b c d 2^{-/-}+\right.$TUDCA $)$ of 18 months of age. Spinal cord immunohistological sections were processed for a-c Iba1, d-f GFAP, $\mathbf{g}-\mathbf{i}$ synaptophysin, $\mathbf{j}-\mathbf{l}$ APP and $\mathbf{m}-\mathbf{o}$ Sudan black. Representative images for WT (a, d, $\mathbf{g}, \mathbf{j}$ and m), $A b c d 1^{-} / A b c d 2^{-I-}(\mathbf{b}$, $\mathbf{e}, \mathbf{h}, \mathbf{k}$, and $\mathbf{n}$ ), and $A b c d 1^{-}$/ $A b c d 2^{-l-}+$ TUDCA (c, $, \mathbf{f}, \mathbf{i}$, $\mathbf{l}$ and $\mathbf{o )}$ mice are shown. Bars $25 \mu \mathrm{m}$. The quantification of synaptophysin (p) and APP (q) in 1-cm-long longitudinal sections of the dorsal spinal cord in WT, $A b c d 1^{-} / A b c d 2^{-/-}$and $A b c d 1^{-} / A b c d 2^{-/-}+$TUDCA mice at $18 \mathrm{~m}$ of age $(n=5$ mice per genotype and condition). The number of abnormal specific profiles was counted at every ten sections for each stain. At least five sections of the spinal cord were analysed per animal and per stain. Clasping (r), treadmill (s) and bar cross (t) tests were conducted on WT, $A b c d 1^{-} / A b c d 2^{-1-}$ and TUDCAtreated $A b c d 1^{-} / A b c d 2^{-/-}$mice (Abcd1 $1^{-} / A b c d 2^{-l-}+$ TUDCA) 17 months of age. $\mathbf{r}$ The best performance score of each animal was used for statistical analysis [19]. $\mathbf{s}$ The latency to falling from the belt (time of shocks), the number of shocks received and the ratio were computed after $5 \mathrm{~min}$. $\mathbf{t}$ The time spent to cross the bar and the numbers of slips of the hind limbs were quantified. Values are expressed as the mean $\pm \mathrm{SD}$ $(n=5$ per condition in $\mathbf{a}-\mathbf{q} ; n=15$ per condition in $\mathbf{r}-\mathbf{t} ; * P<0.05$, $* * P<0.01$ and $* * * P<0.001$, one-way ANOVA followed by Tukey's HSD post hoc test)

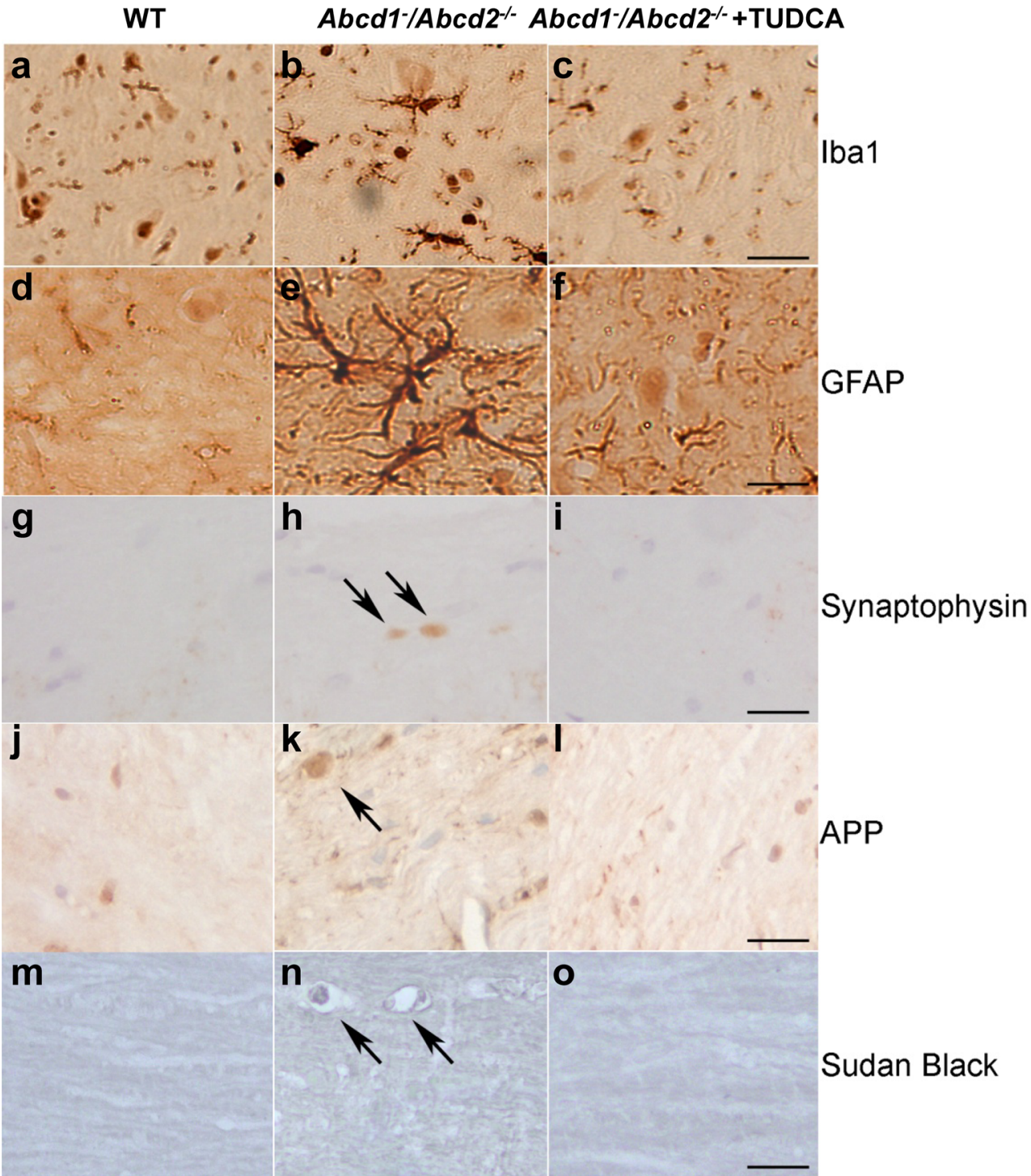

p

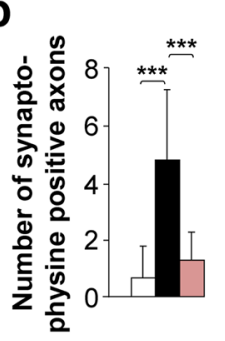

$\mathbf{r}$

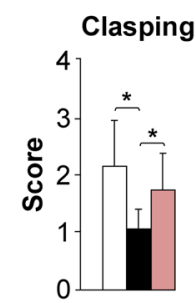

q

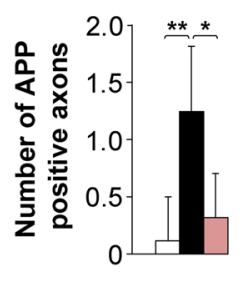

$\square$ WT

Abcd1//Abcd2\%

$\square$ Abcd1//Abcd2 ${ }^{-/}+$TUDCA $\mathbf{s}$

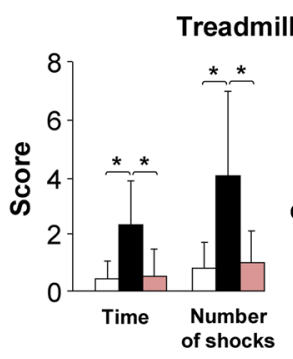

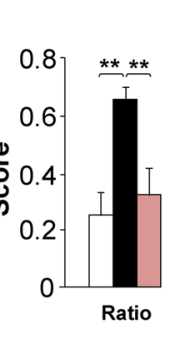

$\mathbf{t}$

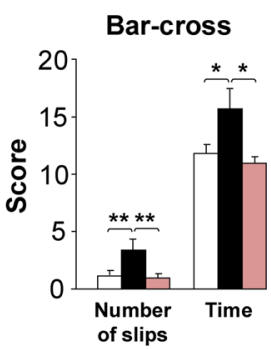




\section{Contribution of peroxisomal malfunction to UPR activation in neurodegenerative disorders}

The ER and peroxisomes share not only a common evolutionary history and ontogeny $[40,84,87]$ but also many intertwined metabolic pathways, particularly the synthesis of the fatty acids that form the lateral chains of complex lipids, such as sphingolipids, phospholipids and plasmalogens, which are main components of membrane lipid bilayers [102]. For instance, the bile acids and DHA are partially synthesized in the ER before entering the peroxisome for the last steps of their synthesis. In addition, VLCFA are elongated from C16:0 in the ER [50, 68], and subsequently imported by $\mathrm{ABCD} 1$ and $\mathrm{ABCD} 2$ transporters into peroxisomes for degradation via $\beta$-oxidation $[30,93]$. The last steps of plasmalogen biosynthesis occur in the ER, but the required intermediates are previously synthesized in the peroxisome [25, 102]. As a consequence, peroxisomal deficiency results in an accumulation of toxic bile acid precursors (tri-hydroxycholestanic acid (THCA) and di-hydroxycholestanic acid (DHCA)) [7, 25] (Supplemental Fig. S6) as well as VLCFA [7], with reduced levels of protective, antioxidant compounds, such as DHA and plasmalogens, in several types of tissues from PBD patients [7].

These observations suggest that UPR activation could be a direct consequence of the unbalanced lipid metabolism that results from peroxisome malfunction.

This is supported by in vivo and in vitro studies that show that the activation of the UPR is directly correlated to a high concentration of saturated phospholipids in ER membranes, in the absence of protein aggregates in the ER lumen, as is the case for the deletion of the mediator subunit mdt-15, a regulator of the transcription of genes involved in fatty acid (FA) metabolism [43]. We thus posit that VLCFA excess may modify the fluidity of the ER lipid bilayer, as shown in model membranes [38, 104]. In X-ALD, this perturbation due to VLCFA excess has recently been speculated to occur in the inner mitochondrial membrane, leading to ROS production [58]. Furthermore, the elongation of VLCFA, which is located in the ER, is increased in X-ALD fibroblasts [50, 68], perhaps contributing to the UPR. In other cases, lipid disequilibrium within the ER membrane could influence the quantity and trafficking of misfolded proteins in the ER lumen, leading indirectly to the activation of UPR transducers [33, 97]. All in all, and in the absence of direct proof of protein aggregates in the ER lumen, we posit that ER membrane lipid perturbation induced by VLCFA accumulation leads to UPR transducer activation, allowing the cell to adapt the ER folding and ERAD machinery igniting an "anticipatory ER stress" response [80, 96] (Fig. 7).

A modification of the ER membrane fluidity by fatty acids is of direct relevance to diseases that are associated with increases in saturated fatty acids, such as non-alcoholic fatty liver [8,77] or type 2 diabetes mellitus [70], which also show ER stress in the absence of misfolded proteins.

Regarding the timing of the observed responses, we observed that in X-ALD and the models of PDB or single peroxisomal enzyme deficiencies, UPR activation occured early in life [23, 45, 53]. In contrast, UPR activation is not an early event in AD [52], Tau disease [39, 52], PD [12, 13] or ALS [51]. We thus hypothesize that late UPR activation, such as that for $\mathrm{AD}$ or PD when compared with that for peroxisomal disorders may be related to the phenomenon of deteriorating peroxisomal fitness over time, as observed during ageing and neurodegenerative disorders $[5,21,32$, $63,89]$. Altogether, these data suggest that an altered peroxisomal function and subsequent lipid metabolism unbalance are key contributors to ER stress, both in rare and common neurodegenerative disorders.

\section{Bile acids in the treatment of neurodegenerative disorders?}

The relevance of bile acids for brain function is illustrated by the observation of large amounts of several intermediates of bile acid biosynthesis in the cerebrospinal fluid [69] (Supplemental Fig. S6). Three of them (glycocholate, glycodeoxycholate, and glycochenodeoxycholate) were elevated in the plasma of AD patients [90], and other compounds of this route were found to be altered in X-ALD adult patients. $5 \beta$-cholestane- $3 \alpha, 7 \alpha, 12 \alpha$-triol, cholate and glycolate are accumulated in the plasma, whereas $5 \beta$-cholestane- $3 \alpha, 7 \alpha, 12 \alpha, 26$-tetrol is increased in peripheral blood mononuclear cells from AMN patients [79]. $P e x 2^{-l-}$ mice also showed an accumulation of the toxic THCA and DHCA in liver tissue, which was concomitant with ER stress $[49,53]$.

UDCA [15] and TUDCA [70, 72] are hydrophilic bile acids that have been used to alleviate ER stress. TUDCA is of particular interest because its levels are lowered in $\mathrm{Pex}^{-/-}$mice [25], thereby suggesting a contribution to ER stress. Indeed, a diet that is enriched in cholic acid and UDCA was able to improve the dendritic arborization of cerebellar Purkinje cells in vivo [22], while also increasing both UDCA and TUDCA levels [49]. Moreover, the chronic administration of UDCA and TUDCA is able to modify the lipid and fatty acid composition of the ER membrane in the rat liver [4]. Treatment with TUDCA has also been shown to ameliorate ER stress in AD [17] and PD mouse models [10]. Altogether, these data suggest that UPR activation may be the result of an alteration in ER membrane fluidity, as noted above, which may be alleviated by replenishing TUDCA levels. We thus posit that levels of bile acids, including TUDCA and fatty acids, are important in ER stress-related disorders and that it is advisable to 


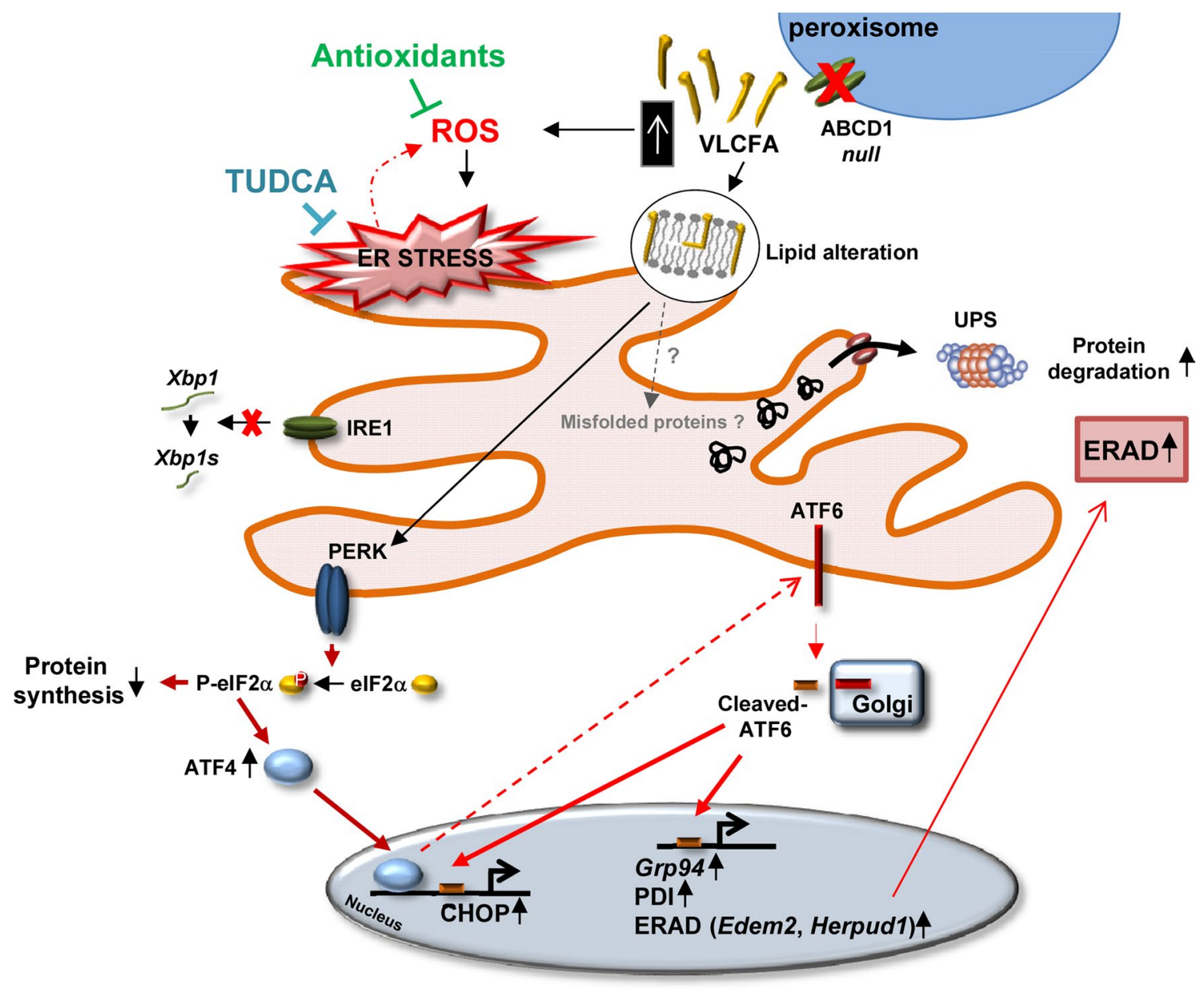

Fig. 7 Mechanisms of UPR activation in the spinal cord from an $\mathrm{X}$-ALD mouse model. Representation of the ER stress response following excess VLCFA due to the lack of ABCD1 function in the $\mathrm{X}$-ALD mouse model. Prior to disease onset (at 12 months), the PERK/P-eIF2 $\alpha /$ ATF4 pathway is activated. It is central for translational control but also for the activation of ATF6 during ER stress, and as a consequence, is critical for the transcription of its target genes, including those involved in protein folding or the ERAD.

include drugs that seek to restore their normal levels when managing these diseases.

Although a systematic comparison of neurodegenerative diseases that highlights therapeutically relevant similarities and differences is still lacking, our data reinforce the notion that ER dysfunction is a pivotal, actionable target for treating neurodegenerative diseases [82]. Interestingly, here we demonstrated that the treatment of X-ALD mice with the ER stress inhibitor TUDCA reduced the progression of axonal degeneration and locomotor disabilities (Fig. 6). This finding is highly relevant because it provides a feasible therapeutic avenue for correcting peroxisomal disorders and neurodegenerative diseases. Indeed, TUDCA has outstanding in vivo safety profiles, is able to cross the blood-brain barrier, and has been approved by the U.S. Food and Drug Administration for
Oxidative stress produced by excess VLCFA and possibly also ER membrane lipid perturbations induced by accumulation of these fatty acids could induce PERK activation independently of unfolded protein formation. This would constitute an adaptive mechanism, allowing the cell to engage ER folding and the ERAD machinery response. Both antioxidant and TUDCA treatments of X-ALD mice prevent ER stress activation and halt subsequent axonal neurodegeneration

clinical use [37]. Clinical trials for ALS and HD have recently been conducted and have yielded promising results regarding safety and efficacy (NCT00877604; NCT00514774) [20, $60,71]$. Thus, we believe that for diseases in which ER stress is an early factor in the pathogenetic cascade, the chances of success may be even greater.

Acknowledgements We are indebted to the NIH NeuroBioBank for supplying the case material used for the human studies. This study was supported by grants from the European Commission [FP7241622], the European Leukodystrophy Association [ELA2012033C1], the Spanish Institute for Health Carlos III and 'Fondo Europeo de Desarrollo Regional (FEDER), Union Europea, una manera de hacer Europa' [FIS PI11/01043, FIS PI14/00410], as well as the Autonomous Government of Catalonia [SGR 2014SGR1430] to A.P., The Spanish Institute for Health Carlos III and 'Fondo Europeo de Desarrollo Regional (FEDER), Union Europea, una manera de 
hacer Europa' [Miguel Servet program CP11/00080, CPII16/00016, FIS PI15/00857] to S.F. and the Center for Biomedical Research on Rare Diseases (CIBERER) to N.L. and M.R. Locomotor experiments were performed by the SEFALer unit F5 led by A.P. which belongs to the CIBERER structure.

\section{Compliance with ethical standards}

Conflict of interest The authors declare that this article was conducted in the absence of any commercial or financial relationships that could be construed as a potential conflict of interest.

Open Access This article is distributed under the terms of the Creative Commons Attribution 4.0 International License (http://creativecommons.org/licenses/by/4.0/), which permits unrestricted use, distribution, and reproduction in any medium, provided you give appropriate credit to the original author(s) and the source, provide a link to the Creative Commons license, and indicate if changes were made.

\section{References}

1. Aubourg P, Blanche S, Jambaque I et al (1990) Reversal of early neurologic and neuroradiologic manifestations of X-linked adrenoleukodystrophy by bone marrow transplantation. N Engl J Med 322:1860-1866

2. Baes M, Van Veldhoven PP (2012) Mouse models for peroxisome biogenesis defects and beta-oxidation enzyme deficiencies. Biochim Biophys Acta 1822:1489-1500

3. Baes M, Van Veldhoven PP (2016) Hepatic dysfunction in peroxisomal disorders. Biochim Biophys Acta 1863(5):956-970

4. Bellentani S, Chao YC, Ferretti I, Panini R, Tiribelli C (1996) Chronic administration of ursodeoxycholic and tauroursodeoxycholic acid changes microsomal membrane lipid content and fatty acid compositions in rats. Biochem Biophys Res Commun 220:479-483

5. Berger J, Dorninger F, Forss-Petter S, Kunze M (2016) Peroxisomes in brain development and function. Biochim Biophys Acta 1863:934-955

6. Berger J, Pujol A, Aubourg P, Forss-Petter S (2010) Current and future pharmacological treatment strategies in X-linked adrenoleukodystrophy. Brain Pathol 20:845-856

7. Braverman NE, Raymond GV, Rizzo WB et al (2016) Peroxisome biogenesis disorders in the Zellweger spectrum: an overview of current diagnosis, clinical manifestations, and treatment guidelines. Mol Genet Metab 117:313-321

8. Cao J, Dai DL, Yao L, Yu HH, Ning B, Zhang Q, Chen J, Cheng WH, Shen W, Yang ZX (2012) Saturated fatty acid induction of endoplasmic reticulum stress and apoptosis in human liver cells via the PERK/ATF4/CHOP signaling pathway. Mol Cell Biochem 364:115-129

9. Cartier N, Hacein-Bey-Abina S, Bartholomae CC et al (2009) Hematopoietic stem cell gene therapy with a lentiviral vector in X-linked adrenoleukodystrophy. Science 326:818-823

10. Castro-Caldas M, Carvalho AN, Rodrigues E, Henderson CJ, Wolf CR, Rodrigues CM, Gama MJ (2012) Tauroursodeoxycholic acid prevents MPTP-induced dopaminergic cell death in a mouse model of Parkinson's disease. Mol Neurobiol 46:475-486

11. Castro RE, Ferreira DM, Zhang X, Borralho PM, Sarver AL, Zeng Y, Steer CJ, Kren BT, Rodrigues CM (2010) Identification of microRNAs during rat liver regeneration after partial hepatectomy and modulation by ursodeoxycholic acid. Am J Physiol Gastrointest Liver Physiol 299:G887-G897
12. Colla E, Coune P, Liu Y, Pletnikova O, Troncoso JC, Iwatsubo T, Schneider BL, Lee MK (2012) Endoplasmic reticulum stress is important for the manifestations of alpha-synucleinopathy in vivo. J Neurosci 32:3306-3320

13. Colla E, Jensen PH, Pletnikova O, Troncoso JC, Glabe C, Lee MK (2012) Accumulation of toxic alpha-synuclein oligomer within endoplasmic reticulum occurs in alpha-synucleinopathy in vivo. J Neurosci 32:3301-3305

14. Crosignani A, Setchell KD, Invernizzi P, Larghi A, Rodrigues CM, Podda M (1996) Clinical pharmacokinetics of therapeutic bile acids. Clin Pharmacokinet 30:333-358

15. Chung J, An SH, Kang SW, Kwon K (2016) Ursodeoxycholic acid (UDCA) exerts anti-atherogenic effects by inhibiting rage signaling in diabetic atherosclerosis. PLoS One 11:e0147839

16. De Munter S, Verheijden S, Regal L, Baes M (2015) Peroxisomal disorders: a review on cerebellar pathologies. Brain Pathol 25:663-678

17. Dionisio PA, Amaral JD, Ribeiro MF, Lo AC, D'Hooge R, Rodrigues CM (2015) Amyloid-beta pathology is attenuated by tauroursodeoxycholic acid treatment in APP/PS1 mice after disease onset. Neurobiol Aging 36:228-240

18. Dirkx R, Vanhorebeek I, Martens K, Schad A, Grabenbauer M, Fahimi D, Declercq P, Van Veldhoven PP, Baes M (2005) Absence of peroxisomes in mouse hepatocytes causes mitochondrial and ER abnormalities. Hepatology 41:868-878

19. Dumser M, Bauer J, Lassmann H, Berger J, Forss-Petter S (2007) Lack of adrenoleukodystrophy protein enhances oligodendrocyte disturbance and microglia activation in mice with combined Abcd1/Mag deficiency. Acta Neuropathol 114:573-586

20. Elia AE, Lalli S, Monsurro MR, Sagnelli A, Taiello AC, Reggiori B, La Bella V, Tedeschi G, Albanese A (2016) Tauroursodeoxycholic acid in the treatment of patients with amyotrophic lateral sclerosis. Eur J Neurol 23(1):45-52

21. Farr RL, Lismont C, Terlecky SR, Fransen M (2016) Peroxisome biogenesis in mammalian cells: the impact of genes and environment. Biochim Biophys Acta 1863:1049-1060

22. Faust PL, Banka D, Siriratsivawong R, Ng VG, Wikander TM (2005) Peroxisome biogenesis disorders: the role of peroxisomes and metabolic dysfunction in developing brain. J Inherit Metab Dis 28:369-383

23. Faust PL, Kovacs WJ (2014) Cholesterol biosynthesis and ER stress in peroxisome deficiency. Biochimie 98:75-85

24. Faust PL, Su HM, Moser A, Moser HW (2001) The peroxisome deficient PEX2 Zellweger mouse: pathologic and biochemical correlates of lipid dysfunction. J Mol Neurosci 16: 289-297 (discussion 317-221)

25. Ferdinandusse S, Denis S, Faust PL, Wanders RJ (2009) Bile acids: the role of peroxisomes. J Lipid Res 50:2139-2147

26. Ferrer I, Aubourg P, Pujol A (2010) General aspects and neuropathology of X-linked adrenoleukodystrophy. Brain Pathol 20:817-830

27. Ferrer I, Kapfhammer JP, Hindelang C et al (2005) Inactivation of the peroxisomal ABCD2 transporter in the mouse leads to late-onset ataxia involving mitochondria, Golgi and endoplasmic reticulum damage. Hum Mol Genet 14:3565-3577

28. Fourcade S, Ferrer I, Pujol A (2015) Oxidative stress, mitochondrial and proteostasis malfunction in adrenoleukodystrophy: a paradigm for axonal degeneration. Free Radic Biol Med 88:18-29

29. Fourcade S, Lopez-Erauskin J, Galino J et al (2008) Early oxidative damage underlying neurodegeneration in X-adrenoleukodystrophy. Hum Mol Genet 17:1762-1773

30. Fourcade S, Ruiz M, Camps C et al (2009) A key role for the peroxisomal ABCD2 transporter in fatty acid homeostasis. Am J Physiol Endocrinol Metab 296:E211-E221 
31. Fourcade S, Ruiz M, Guilera C et al (2010) Valproic acid induces antioxidant effects in X-linked adrenoleukodystrophy. Hum Mol Genet 19:2005-2014

32. Fransen M, Nordgren M, Wang B, Apanasets O, Van Veldhoven PP (2013) Aging, age-related diseases and peroxisomes. Subcell Biochem 69:45-65

33. Fu S, Yang L, Li P, Hofmann O, Dicker L, Hide W, Lin X, Watkins SM, Ivanov AR, Hotamisligil GS (2011) Aberrant lipid metabolism disrupts calcium homeostasis causing liver endoplasmic reticulum stress in obesity. Nature 473:528-531

34. Galino J, Ruiz M, Fourcade S et al (2011) Oxidative damage compromises energy metabolism in the axonal degeneration mouse model of x-adrenoleukodystrophy. Antioxid Redox Signal 15:2095-2107

35. Hein S, Schonfeld P, Kahlert S, Reiser G (2008) Toxic effects of X-linked adrenoleukodystrophy-associated, very long chain fatty acids on glial cells and neurons from rat hippocampus in culture. Hum Mol Genet 17:1750-1761

36. Hetz C, Mollereau B (2014) Disturbance of endoplasmic reticulum proteostasis in neurodegenerative diseases. Nat Rev Neurosci 15:233-249

37. Heubi JE, Wiechmann DA, Creutzinger V, Setchell KD, Squires R Jr, Couser R, Rhodes P (2002) Tauroursodeoxycholic acid (TUDCA) in the prevention of total parenteral nutrition-associated liver disease. J Pediatr 141:237-242

38. Ho JK, Moser H, Kishimoto Y, Hamilton JA (1995) Interactions of a very long chain fatty acid with model membranes and serum albumin. Implications for the pathogenesis of adrenoleukodystrophy. J Clin Invest 96:1455-1463

39. Ho YS, Yang X, Lau JC, Hung CH, Wuwongse S, Zhang Q, Wang J, Baum L, So KF, Chang RC (2012) Endoplasmic reticulum stress induces tau pathology and forms a vicious cycle: implication in Alzheimer's disease pathogenesis. J Alzheimers Dis $28: 839-854$

40. Hoepfner D, Schildknegt D, Braakman I, Philippsen P, Tabak HF (2005) Contribution of the endoplasmic reticulum to peroxisome formation. Cell 122:85-95

41. Hoozemans JJ, van Haastert ES, Nijholt DA, Rozemuller AJ, Eikelenboom P, Scheper W (2009) The unfolded protein response is activated in pretangle neurons in Alzheimer's disease hippocampus. Am J Pathol 174:1241-1251

42. Hoozemans JJ, Veerhuis R, Van Haastert ES, Rozemuller JM, Baas F, Eikelenboom P, Scheper W (2005) The unfolded protein response is activated in Alzheimer's disease. Acta Neuropathol 110:165-172

43. Hou NS, Gutschmidt A, Choi DY, Pather K, Shi X, Watts JL, Hoppe T, Taubert S (2014) Activation of the endoplasmic reticulum unfolded protein response by lipid disequilibrium without disturbed proteostasis in vivo. Proc Natl Acad Sci 111:E2271-E2280

44. Hu Y, Park KK, Yang L et al (2011) Differential effects of unfolded protein response pathways on axon injury-induced death of retinal ganglion cells. Neuron 73:445-452

45. Huang J, Viswakarma N, Yu S et al (2011) Progressive endoplasmic reticulum stress contributes to hepatocarcinogenesis in fatty acyl-CoA oxidase 1-deficient mice. Am J Pathol 179:703-713

46. Ilieva EV, Kichev A, Naudi A, Ferrer I, Pamplona R, PorteroOtin M (2011) Mitochondrial dysfunction and oxidative and endoplasmic reticulum stress in argyrophilic grain disease. $\mathrm{J}$ Neuropathol Exp Neurol 70:253-263

47. Ilieva EV, Naudi A, Kichev A, Ferrer I, Pamplona R, PorteroOtin M (2010) Depletion of oxidative and endoplasmic reticulum stress regulators in Pick disease. Free Radic Biol Med 48:1302-1310

48. Infante JP, Huszagh VA (2001) Zellweger syndrome knockout mouse models challenge putative peroxisomal beta-oxidation involvement in docosahexaenoic acid (22:6n-3) biosynthesis. Mol Genet Metab 72:1-7

49. Keane MH, Overmars H, Wikander TM, Ferdinandusse S, Duran M, Wanders RJ, Faust PL (2007) Bile acid treatment alters hepatic disease and bile acid transport in peroxisomedeficient PEX2 Zellweger mice. Hepatology 45:982-997

50. Kemp S, Valianpour F, Denis S, Ofman R, Sanders RJ, Mooyer P, Barth PG, Wanders RJ (2005) Elongation of very long-chain fatty acids is enhanced in X-linked adrenoleukodystrophy. Mol Genet Metab 84:144-151

51. Kikuchi H, Almer G, Yamashita S, Guegan C, Nagai M, Xu Z, Sosunov AA, McKhann GM 2nd, Przedborski S (2006) Spinal cord endoplasmic reticulum stress associated with a microsomal accumulation of mutant superoxide dismutase-1 in an ALS model. Proc Natl Acad Sci 103:6025-6030

52. Kohler C, Dinekov M, Gotz J (2014) Granulovacuolar degeneration and unfolded protein response in mouse models of tauopathy and Abeta amyloidosis. Neurobiol Dis 71:169-179

53. Kovacs WJ, Charles KN, Walter KM, Shackelford JE, Wikander TM, Richards MJ, Fliesler SJ, Krisans SK, Faust PL (2012) Peroxisome deficiency-induced ER stress and SREBP-2 pathway activation in the liver of newborn PEX2 knock-out mice. Biochim Biophys Acta 1821:895-907

54. Kovacs WJ, Tape KN, Shackelford JE, Wikander TM, Richards MJ, Fliesler SJ, Krisans SK, Faust PL (2009) Peroxisome deficiency causes a complex phenotype because of hepatic SREBP/ Insig dysregulation associated with endoplasmic reticulum stress. J Biol Chem 284:7232-7245

55. Launay N, Aguado C, Fourcade S et al (2015) Autophagy induction halts axonal degeneration in a mouse model of X-adrenoleukodystrophy. Acta Neuropathol 129:399-415

56. Launay N, Ruiz M, Fourcade S, Schluter A, Guilera C, Ferrer I, Knecht E, Pujol A (2013) Oxidative stress regulates the ubiquitin-proteasome system and immunoproteasome functioning in a mouse model of $\mathrm{X}$-adrenoleukodystrophy. Brain 136:891-904

57. Lopez-Erauskin J, Fourcade S, Galino J et al (2011) Antioxidants halt axonal degeneration in a mouse model of X-adrenoleukodystrophy. Ann Neurol 70:84-92

58. Lopez-Erauskin J, Galino J, Ruiz M et al (2013) Impaired mitochondrial oxidative phosphorylation in the peroxisomal disease X-linked adrenoleukodystrophy. Hum Mol Genet 22:3296-3305

59. Lu JF, Lawler AM, Watkins PA, Powers JM, Moser AB, Moser HW, Smith KD (1997) A mouse model for X-linked adrenoleukodystrophy. Proc Natl Acad Sci 94:9366-9371

60. Ludolph AC (2015) The TUDCA trial-innovative trial designs for amyotrophic lateral sclerosis drugs? Eur J Neurol 23:11-12

61. Malhotra JD, Kaufman RJ (2007) Endoplasmic reticulum stress and oxidative stress: a vicious cycle or a double-edged sword? Antioxid Redox Signal 9:2277-2293

62. Mastroeni R, Bensadoun JC, Charvin D, Aebischer P, Pujol A, Raoul C (2009) Insulin-like growth factor-1 and neurotrophin-3 gene therapy prevents motor decline in an X-linked adrenoleukodystrophy mouse model. Ann Neurol 66:117-122

63. Mielke MM, Maetzler W, Haughey NJ et al (2013) Plasma ceramide and glucosylceramide metabolism is altered in sporadic Parkinson's disease and associated with cognitive impairment: a pilot study. PLoS One 8:e73094

64. Morato L, Galino J, Ruiz M et al (2013) Pioglitazone halts axonal degeneration in a mouse model of X-linked adrenoleukodystrophy. Brain 136:2432-2443

65. Morato L, Ruiz M, Boada J et al (2015) Activation of sirtuin 1 as therapy for the peroxisomal disease adrenoleukodystrophy. Cell Death Differ 22:1742-1753

66. Moser H, Smith, KD, Watkins, PA, Powers, J, Moser, AB (2001) X-linked adrenoleukodystrophy. In: Scriver C (ed) The 
metabolic and molecular bases of inherited disease, 8th edn. McGraw-Hill, New York, pp 3257-3301

67. Nunes AF, Amaral JD, Lo AC, Fonseca MB, Viana RJ, Callaerts-Vegh Z, D'Hooge R, Rodrigues CM (2012) TUDCA, a Bile acid, attenuates amyloid precursor protein processing and amyloid-beta deposition in APP/PS1 mice. Mol Neurobiol 45:440-454

68. Ofman R, Dijkstra IM, van Roermund CW, Burger N, Turkenburg M, van Cruchten A, van Engen CE, Wanders RJ, Kemp S (2010) The role of ELOVL1 in very long-chain fatty acid homeostasis and X-linked adrenoleukodystrophy. EMBO Mol Med 2:90-97

69. Ogundare M, Theofilopoulos S, Lockhart A, Hall LJ, Arenas E, Sjovall J, Brenton AG, Wang Y, Griffiths WJ (2010) Cerebrospinal fluid steroidomics: are bioactive bile acids present in brain? J Biol Chem 285:4666-4679

70. Ozcan L, Ergin AS, Lu A, Chung J, Sarkar S, Nie D, Myers MG Jr, Ozcan U (2009) Endoplasmic reticulum stress plays a central role in development of leptin resistance. Cell Metab 9:35-51

71. Parry GJ, Rodrigues CM, Aranha MM, Hilbert SJ, Davey C, Kelkar P, Low WC, Steer CJ (2010) Safety, tolerability, and cerebrospinal fluid penetration of ursodeoxycholic acid in patients with amyotrophic lateral sclerosis. Clin Neuropharmacol 33:17-21

72. Perlmutter DH (2002) The cellular response to aggregated proteins associated with human disease. J Clin Invest 110:1219-1220

73. Petrillo S, Piemonte F, Pastore A, Tozzi G, Aiello C, Pujol A, Cappa M, Bertini E (2013) Glutathione imbalance in patients with X-linked adrenoleukodystrophy. Mol Genet Metab 109:366-370

74. Powers JM, Pei Z, Heinzer AK, Deering R, Moser AB, Moser HW, Watkins PA, Smith KD (2005) Adreno-leukodystrophy: oxidative stress of mice and men. J Neuropathol Exp Neurol 64:1067-1079

75. Pujol A, Ferrer I, Camps C, Metzger E, Hindelang C, Callizot N, Ruiz M, Pampols T, Giros M, Mandel JL (2004) Functional overlap between ABCD1 (ALD) and ABCD2 (ALDR) transporters: a therapeutic target for X-adrenoleukodystrophy. Hum Mol Genet 13:2997-3006

76. Pujol A, Hindelang C, Callizot N, Bartsch U, Schachner M, Mandel JL (2002) Late onset neurological phenotype of the $\mathrm{X}$-ALD gene inactivation in mice: a mouse model for adrenomyeloneuropathy. Hum Mol Genet 11:499-505

77. Puri P, Mirshahi F, Cheung O, Natarajan R, Maher JW, Kellum JM, Sanyal AJ (2008) Activation and dysregulation of the unfolded protein response in nonalcoholic fatty liver disease. Gastroenterology 134:568-576

78. Roselli F, Caroni P (2012) Life-or-death decisions upon axonal damage. Neuron 73:405-407

79. Ruiz M, Jove M, Schluter A et al (2015) Altered glycolipid and glycerophospholipid signaling drive inflammatory cascades in adrenomyeloneuropathy. Hum Mol Genet 24:6861-6876

80. Rutkowski DT, Hegde RS (2010) Regulation of basal cellular physiology by the homeostatic unfolded protein response. J Cell Biol 189:783-794

81. Sasaki S (2010) Endoplasmic reticulum stress in motor neurons of the spinal cord in sporadic amyotrophic lateral sclerosis. J Neuropathol Exp Neurol 69:346-355

82. Scheper W, Hoozemans JJ (2015) The unfolded protein response in neurodegenerative diseases: a neuropathological perspective. Acta Neuropathol 130:315-331

83. Schluter A, Fourcade S, Domenech-Estevez E, Gabaldon T, Huerta-Cepas J, Berthommier G, Ripp R, Wanders RJ, Poch O, Pujol A (2007) PeroxisomeDB: a database for the peroxisomal proteome, functional genomics and disease. Nucleic Acids Res 35:D815-D822

84. Schluter A, Fourcade S, Ripp R, Mandel JL, Poch O, Pujol A (2006) The Evolutionary origin of peroxisomes: an ER-peroxisome connection. Mol Biol Evol 23:838-845

85. Singh I, Pujol A (2010) Pathomechanisms underlying X-adrenoleukodystrophy: a three-hit hypothesis. Brain Pathol 20:838-844

86. Southwood CM, Garbern J, Jiang W, Gow A (2002) The unfolded protein response modulates disease severity in Pelizaeus-Merzbacher disease. Neuron 36:585-596

87. Tabak HF, Braakman I, van der Zand A (2013) Peroxisome formation and maintenance are dependent on the endoplasmic reticulum. Annu Rev Biochem 82:723-744

88. Teske BF, Wek SA, Bunpo P, Cundiff JK, McClintick JN, Anthony TG, Wek RC (2011) The eIF2 kinase PERK and the integrated stress response facilitate activation of ATF6 during endoplasmic reticulum stress. Mol Biol Cell 22:4390-4405

89. Trompier D, Vejux A, Zarrouk A, Gondcaille C, Geillon F, Nury T, Savary S, Lizard G (2014) Brain peroxisomes. Biochimie 98:102-110

90. Trushina E, Mielke MM (2014) Recent advances in the application of metabolomics to Alzheimer's Disease. Biochim Biophys Acta 1842:1232-1239

91. van der Vlies D, Makkinje M, Jansens A, Braakman I, Verkleij AJ, Wirtz KW, Post JA (2003) Oxidation of ER resident proteins upon oxidative stress: effects of altering cellular redox/ antioxidant status and implications for protein maturation. Antioxid Redox Signal 5:381-387

92. van Kollenburg B, van Dijk J, Garbern J, Thomas AA, Scheper GC, Powers JM, van der Knaap MS (2006) Glia-specific activation of all pathways of the unfolded protein response in vanishing white matter disease. J Neuropathol Exp Neurol 65:707-715

93. van Roermund CW, Visser WF, Ijlst L, van Cruchten A, Boek M, Kulik W, Waterham HR, Wanders RJ (2008) The human peroxisomal ABC half transporter ALDP functions as a homodimer and accepts acyl-CoA esters. Faseb J 22:4201-4208

94. Vargas CR, Wajner M, Sirtori LR et al (2004) Evidence that oxidative stress is increased in patients with X-linked adrenoleukodystrophy. Biochim Biophys Acta 1688:26-32

95. Vidal RL, Figueroa A, Court FA et al (2012) Targeting the UPR transcription factor XBP1 protects against Huntington's disease through the regulation of FoxO1 and autophagy. Hum Mol Genet 21:2245-2262

96. Vitale A, Boston RS (2008) Endoplasmic reticulum quality control and the unfolded protein response: insights from plants. Traffic 9:1581-1588

97. Volmer R, Ron D (2015) Lipid-dependent regulation of the unfolded protein response. Curr Opin Cell Biol 33:67-73

98. Volmer R, van der Ploeg K, Ron D (2013) Membrane lipid saturation activates endoplasmic reticulum unfolded protein response transducers through their transmembrane domains. Proc Natl Acad Sci 110:4628-4633

99. Walter KM, Schonenberger MJ, Trotzmuller M et al (2014) Hif2alpha promotes degradation of mammalian peroxisomes by selective autophagy. Cell Metab 20:882-897

100. Walter P, Ron D (2011) The unfolded protein response: from stress pathway to homeostatic regulation. Science 334:1081-1086

101. Wanders RJ, van Roermund CW, van Wijland MJ, Schutgens RB, Schram AW, Tager JM, van den Bosch H, Schalkwijk C (1988) X-linked adrenoleukodystrophy: identification of the primary defect at the level of a deficient peroxisomal very long chain fatty acyl-CoA synthetase using a newly developed 
method for the isolation of peroxisomes from skin fibroblasts. $\mathbf{J}$ Inherit Metab Dis 11(Suppl 2):173-177

102. Wanders RJ, Waterham HR, Ferdinandusse S (2016) Metabolic interplay between peroxisomes and other subcellular organelles including mitochondria and the endoplasmic reticulum. Front Cell Dev Biol 3:83

103. Waterham HR, Ferdinandusse S, Wanders RJ (2016) Human disorders of peroxisome metabolism and biogenesis. Biochim Biophys Acta 1863:922-933

104. Whitcomb RW, Linehan WM, Knazek RA (1988) Effects of long-chain, saturated fatty acids on membrane microviscosity and adrenocorticotropin responsiveness of human adrenocortical cells in vitro. J Clin Invest 81:185-188

105. Ye J, Rawson RB, Komuro R, Chen X, Dave UP, Prywes R, Brown MS, Goldstein JL (2000) ER stress induces cleavage of membrane-bound ATF6 by the same proteases that process SREBPs. Mol Cell 6:1355-1364

106. Zhang YJ, Jansen-West K, Xu YF et al (2014) Aggregationprone c9FTD/ALS poly(GA) RAN-translated proteins cause neurotoxicity by inducing ER stress. Acta Neuropathol 128:505-524 